\title{
Spanwise structuring and rivulet formation in suspended falling liquid films
}

\author{
Manuel Rietz $\odot^{*}$ and Reinhold Kneer®e \\ Institute of Heat and Mass Transfer, RWTH Aachen University, Augustinerbach 6, 52056 Aachen, Germany \\ Benoit Scheid \\ TIPs, Université libre de Bruxelles, Avenue Franklin D. Roosevelt 50, 1050 Brussels, Belgium \\ Wilko Rohlfs $\odot$ \\ Department of Thermal and Fluid Engineering, University of Twente, Drienerlolaan 5, 7522 NB Enschede, \\ The Netherlands
}

(Received 23 November 2020; accepted 15 July 2021; published 25 August 2021)

\begin{abstract}
In suspended falling films, i.e., films on the underside of a bounding wall with arbitrary inclination, the surface film topology evolves towards a distinct spanwise structuring of the flow into rivulets, which is potentially accompanied by dripping events. Experimental data suggest a connection between long-term spanwise structuring and primary instabilities of the film surface. However, available experimental data regarding this connection remain nonconclusive. Hence, the present study aims at elucidating the evolution of a suspended falling film from varying imposed initial conditions to the emergence of spanwise modulations and rivulet formation. The study is carried out by means of extended numerical simulations employing a weighted residual integral boundary layer model for falling liquid films. The investigated parameter space covers recent experimental data on the topic. Varying imposed initial conditions, Reynolds number, Kapitza number, as well as wall inclination, several possible causes for a deviation of observed spanwise wavelengths from the one predicted by the primary Rayleigh-Taylor mechanism, are identified. This includes a distinct influence of initial conditions, asynchronous destabilization of consecutive wavefronts, competing short wave capillary instabilities, and rivulet interaction.
\end{abstract}

DOI: 10.1103/PhysRevFluids.6.084805

\section{INTRODUCTION}

In falling liquid films a cascade of primary and secondary fluid dynamical instabilities leads to complex patterns of waves on the film surface as well as characteristic vortex structures in the liquid and surrounding gaseous phase. These flow structures are associated with an increase in surface area and result in favorable heat transfer rates or analogously mass transfer rates in multicomponent solutions [1-3]. Not surprisingly, falling films are encountered in various technical systems in energy and process engineering, from heat exchangers and distillation processes to nuclear fusion reactor design [4-6] or coating processes and the fabrication of polymer shells [7,8].

In the special case of a film flow on the underside of a substrate (suspended film) with sufficient inclination from the vertical, dripping, i.e., fluid detachment from the substrate, might occur [9-11]. The fluid dynamical instability associated with dripping is the Rayleigh-Taylor (RT) instability [12-14], which arises if a denser fluid (film) is accelerated towards a less dense fluid (ambient gas), for example, through gravitational acceleration [15] or centrifugal acceleration [16] or even

*rietz@wsa.rwth-aachen.de 
through electric forces [17]. Reinforcement or prevention of dripping is of significant importance in many engineering situations. In coating problems, a destabilization of the film might lead to undesired inhomogeneities of the surface topology. In fact, several recent studies have explored the suppression of the RT instability through, e.g., vibration of the substrate [18], thermal gradients at the film surface [19], surface tension gradients [20], or the application of electric fields [21,22]. At the same time, Mathelot et al. [23] have demonstrated that the RT instability acting on a curing polymer film on a rotating cylinder can be "harvested" to create soft materials with regularly structured surfaces over many length scales. In cooling tower fills or structured packings in chemical engineering, dripping leads to increased surface areas for transfer processes, but at the same time to decreased residence times within the system, leaving room for potential optimization.

Experimental studies of suspended falling films or more general films under the influence of a destabilizing body force have demonstrated that dripping is generally preceded by a distinct spanwise structuring of the flow into rivulets $[16,24,25]$. However, only a few studies have analyzed the wavelength of developing rivulet structures, let alone its evolution over time. For a low Reynolds number $(\mathrm{Re} \lesssim 1)$ falling liquid film, characterized by a low Kapitza number, $\mathrm{Ka}=5.1$, it has been shown that the wavelength of evolving rivulets can be predicted by a wall inclination angledependent formulation of the RT mechanism with satisfying accuracy [16]. Ledda et al. [26] and Lerisson et al. [27] have experimentally and numerically investigated the emergence and stability of rivulet structures putting in evidence a secondary instability leading to traveling lenses emerging on top of these streamwise-aligned structures. Experimental data, in this context, have been presented for a viscous fluid and negligible inertia. Again, a wall inclination angle-dependent formulation of the RT mechanism was found to describe the wavelength of emerging rivulets. At the same time, a set of experimental data on the topic provided by the study of Charogiannis et al. [11] points to the wavelength selection mechanism being more intricate for increased Reynolds and Kapitza numbers. Specifically, it was observed that the measured rivulet wavelength either coincides with the one predicted by the RT mechanism under consideration of the angle of inclination or under neglecting the angle of inclination. The latter equals the classical formulation of the RT wavelength for a fluid layer beneath a horizontal wall. A dependency on imposed flow rate and inclination angle was shown. However, the physical explanation for the experimentally observed effect as well as possible influences of imposed initial conditions remains unclear. At the same time, a thorough description of the evolution of rivulet wavelengths in suspended falling films has important implications for a correct interpretation of related experimental and numerical investigations. In an experiment, the length of the experimental test section as well as the measurement position have to be chosen accordingly to the state of the evolution of the falling film, which is of interest to the respective study. Hence, a potentially complex evolution of rivulet structures has to be taken into account. In numerical investigations, awareness of the consequences of the choice of initial condition and imposed noise on rivulet evolution are key in reproducing realistic results, which might be observed in real-world applications. In the end, a complex wavelength selection mechanism of evolving rivulets is supposed to have a major influence on the dripping regime and its prediction as well as reinforcement or suppression.

The present study aims at elucidating the evolution of a suspended falling film from varying imposed initial conditions to the emergence of quasisteady rivulet structures. In this context, specific focus lies on the evolution of the number of rivulets (their wavelength) over time. To this end, numerical simulations employing the well-established weighted residual integral boundary layer (WRIBL) model for falling liquid films are performed in an extended parameter space of Re and $\mathrm{Ka}$, which includes recent experimental data on the topic [11]. The purpose of this numerical study is twofold. First, it is examined under which circumstances and to which extent spanwise structuring of suspended falling films can be rationalized by predictions made by an angle-dependent formulation of the RT mechanism. Second, additional effects which lead to an increase in complexity regarding rivulet formation and evolution are described and analyzed. This includes the influence of the chosen initial condition, wave-to-wave interactions, and capillary instabilities. Finally, we rationalize the various observations on rivulet wavelengths observed in experiments. 


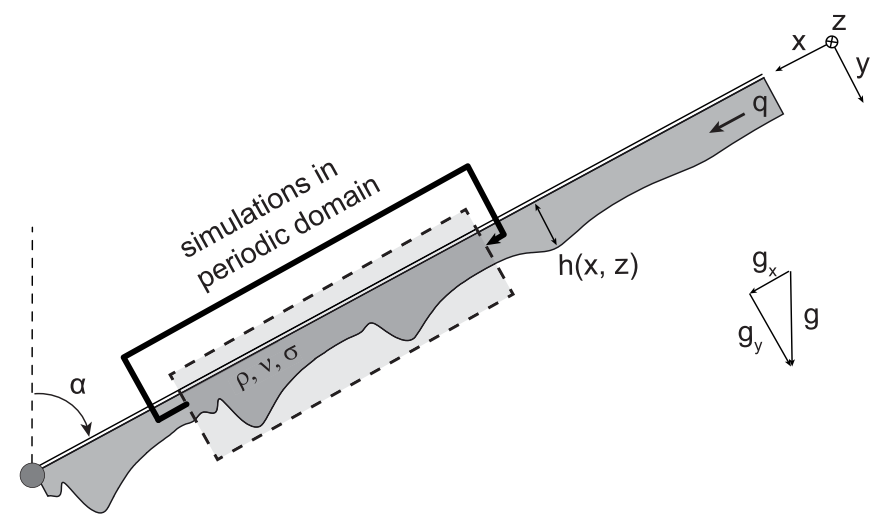

FIG. 1. Schematic of a suspended falling liquid film under the destabilizing action of gravity, with inclination $\alpha$ from the vertical. Simulations are performed in a domain with periodic boundary conditions in the $x$-direction and $z$-direction.

The paper is organized as follows. In Sec. II the governing equations and boundary conditions are recalled briefly. The third section outlines the numerical procedure and provides a discussion of domain size, resolution, and the choice of the initial condition. In the results section, spanwise structuring and rivulet formation are discussed in detail for two specific flow conditions: a low Reynolds and low Kapitza number case and a case with moderate Reynolds and Kapitza numbers. In both cases, the inclination angle is varied to provide insights into the mechanisms of rivulet formation and evolution. The paper ends with a parametric study, varying the Kapitza number in the range defined by the two previously introduced cases.

\section{GOVERNING EQUATIONS AND BOUNDARY CONDITIONS}

The relevant physical parameters in dimensionless space characterizing an isothermal film flow of Newtonian fluid under a tilted plane wall consist of Reynolds number (Re), Kapitza number (Ka), and inclination number $(\mathrm{Ct})$ :

$$
\mathrm{Re}=\frac{q}{v}, \quad \mathrm{Ka}=\frac{\sigma}{\rho g_{x}^{1 / 3} v^{4 / 3}}, \quad \mathrm{Ct}=-\frac{g_{\mathrm{y}}}{g_{\mathrm{x}}}=-\frac{\sin \alpha}{\cos \alpha},
$$

with $q$, the flow rate per unit length, $v$, the fluid kinematic viscosity, $\sigma$, the fluid surface tension, $\rho$, the fluid density, $g_{x}$, the streamwise component of gravitational acceleration, $g_{y}$, the wall normal component of gravitational acceleration, and $\alpha$, the wall inclination from the vertical. The inclination number is well defined by the limiting cases of wall inclination, namely, a vertical and a horizontal wall, corresponding to inclination numbers zero and minus infinity. The Weber number (We) can be expressed through Kapitza and Reynolds numbers by

$$
\mathrm{We}=\frac{\mathrm{Ka}}{(3 \mathrm{Re})^{2 / 3}} .
$$

The boundary layer approximation for the problem in question is obtained through introduction of an ordering parameter $\epsilon$, which compares within the film weak gradients in streamwise and spanwise directions, to strong gradients in the cross-stream direction, such that $\epsilon \ll 1$. The derivation of the boundary layer equations was thoroughly introduced, for example, by Kalliadasis et al. [3]. Thus, only the respective system of dimensionless conservation equations and boundary conditions for the investigated case of an isothermal suspended falling film of Newtonian fluid is given here. A Cartesian coordinate system $(x, y, z)$ is introduced denoting the streamwise, crosswise, and spanwise coordinates of the falling film (see Fig. 1) together with a respective velocity vector $(u, v, w)$. The 
position of the wall is fixed at $y=0$. The position of the interface, $h$, is a function of space and time, $h=f(x, z, t)$.

Employing the given definitions, the continuity equation reads

$$
\partial_{x} u+\partial_{y} v+\partial_{z} w=0
$$

The equations for streamwise, crosswise, and spanwise momentum conservation are given by

$$
\begin{gathered}
3 \epsilon \operatorname{Re}\left(\partial_{t} u+u \partial_{x} u+v \partial_{y} u+w \partial_{z} u\right)=-\epsilon \partial_{x} p+\epsilon^{2} \partial_{x x} u+\partial_{y y} u+\epsilon^{2} \partial_{z z} u+1, \\
3 \epsilon^{2} \operatorname{Re}\left(\partial_{t} v+u \partial_{x} v+v \partial_{y} v+w \partial_{z} v\right)=-\partial_{y} p+\epsilon \partial_{y y} v-\mathrm{Ct}, \\
3 \epsilon \operatorname{Re}\left(\partial_{t} w+u \partial_{x} w+v \partial_{y} w+w \partial_{z} w\right)=-\partial_{z} p+\epsilon^{2} \partial_{x x} w+\partial_{y y} w+\epsilon^{2} \partial_{z z} w,
\end{gathered}
$$

respectively. Note that terms which are of order $\epsilon^{3}$ have been neglected. The dimensionless boundary condition at the wall $(y=0)$ is given by

$$
u=v=w=0 .
$$

At the free surface $h(x, z, t)$, the kinematic boundary condition is given by

$$
v=\partial_{t} h+u \partial_{x} h+w \partial_{z} h .
$$

Additionally, dynamic boundary conditions at the free surface describe the normal and the two tangential stress balances in the form

$$
\begin{aligned}
& p=2 \epsilon\left(\partial_{y} v-\partial_{x} h \partial_{y} u-\partial_{z} h \partial_{y} u\right)-\epsilon^{2} \operatorname{We}\left(\partial_{x x} h+\partial_{z z} h\right), \\
& \partial_{y} u=\epsilon^{2}\left[\partial_{z} h\left(\partial_{z} u+\partial_{x} w\right)+2 \partial_{x} h\left(2 \partial_{x} u+\partial_{z} w\right)-\partial_{x} v\right], \\
& \partial_{y} w=\epsilon^{2}\left[\partial_{x} h\left(\partial_{z} u+\partial_{x} w\right)+2 \partial_{z} h\left(2 \partial_{z} u+\partial_{x} w\right)-\partial_{z} v\right],
\end{aligned}
$$

in which terms of order $\epsilon^{3}$ have been neglected.

\section{NUMERICAL PROCEDURE}

The numerical analysis of spanwise structuring and rivulet formation in suspended falling films poses specific requirements to the efficiency of the employed simulation method. This is because either (1) long domains or (2) large simulation times in combination with periodic boundary conditions are required to capture the entire film evolution until a characteristic quasistationary film topology develops. Even though the results of both approaches are not strictly identical, long evolution times or lengths hold especially for low inclinations from the vertical, where growth rates of perturbations are generally low compared to the flow rate. Fully resolved numerical simulations are hardly applicable in this context [28]. Thus, models of reduced dimensionality are the method of choice. The weighted residuals integral boundary layer model of Ruyer-Quil and Manneville [29] (WRIBL model) is employed here to allow for long-term simulations with a manageable amount of computational cost. Derivation of the full second-order 2D WRIBL model obtained from the system of Eqs. (1)-(9) was introduced in Scheid et al. [30]. This model consists of a set of seven coupled evolution equations for the film thickness $h$, the flow rate components $q_{\|}$and $q_{\perp}$ in the streamwise and spanwise directions, and two corrections for each flow rate component, accounting for secondorder inertia effects. An efficient computational implementation of the model has recently been provided by Rohlfs et al. [31] in the form of the open access software package WAVEMAKER. In WAVEMAKER, the model is solved using a pseudospectral method. Hence, simulations are performed in a domain with periodic boundary conditions in streamwise and spanwise directions. 
TABLE I. Fluid properties and simulation parameters.

\begin{tabular}{lccc}
\hline \hline & Case I & Case II & \\
\hline$\rho$ & 1214 & 1127 & {$\left[\mathrm{~kg} / \mathrm{m}^{3}\right]$} \\
$v$ & 49.9 & 4.4 & {$\left[10^{-6} \mathrm{~m}^{2} / \mathrm{s}\right]$} \\
$\sigma$ & 62.4 & 57.7 & {$\left[10^{-3} \mathrm{~N} / \mathrm{m}\right]$} \\
$l_{c}$ & 2.285 & {$\left[10^{-3} \mathrm{~m}\right]$} \\
$\operatorname{Re}$ & 2.289 & 40 & - \\
$\mathrm{Ka}_{\alpha=0^{\circ}}$ & 1 & 330 & - \\
$\mathrm{Ct}$ & 13.1 & $0-3.72$ & - \\
$\alpha$ & $0-3.72$ & $0-75$ & {$[\mathrm{deg}]$} \\
$\alpha_{c}[10]$ & $0-75$ & 85 & {$[\mathrm{deg}]$} \\
\hline \hline
\end{tabular}

\section{A. Simulation parameters}

Fluid properties as well as the parameter space for the simulations are defined in Table I. In this context, the properties of two different aqueous-glycerol solutions are employed corresponding to the fluids used in the experimental study by Charogiannis et al. [11]. The two cases comprise a low Reynolds and Kapitza numbers case (case I) and an intermediate Reynolds and Kapitza numbers case (case II), both lying within the validity range of the employed numerical model [3]. The choice of these fluids for the simulations has several advantages: (1) The most obvious advantage is that experimental data are available for a part of the parameter space (case I: $\alpha=30^{\circ}$, case II: $\alpha=$ $\left.\left[15^{\circ}, 30^{\circ}, 45^{\circ}\right][11]\right)$ allowing for a comparison to simulation results. (2) A less obvious advantage is that the two fluids, significantly differing only in their viscosity, have a comparable capillary length defined as

$$
l_{c}=\sqrt{\frac{\sigma}{\rho g}},
$$

which scales the most amplified wavelength of the RT instability [see Eq. (15)]. Consequently, almost identical capillary lengths enable direct comparability of the cases with respect to expected and observed spanwise structuring. The Reynolds number is fixed for each fluid independently of the wall inclination, which can be associated with an increasing film thickness with increasing inclination from the vertical. At the same time, the vertical Kapitza number $\mathrm{Ka}_{\alpha=0^{\circ}}$ is fixed by the respective fluid properties. Simulations have been performed with inclination numbers ranging from 0 to 3.72 , corresponding to wall inclinations between $0^{\circ}$ and $75^{\circ}$ from the vertical. Angles closer to the horizontal have not been investigated due to the tendency of the film flow to drip after short times. Dripping as such cannot be resolved by the WRIBL model being a single-value model and thus leads to model failure. An estimate of the critical inclination angle $\alpha_{c}$ below which the flow is always linearly convectively unstable and hence immediate dripping is not expected was provided by Scheid et al. [10] based on the linear transition between absolute and convective instability. The values of $\alpha_{c}$ for cases I and II are given in Table I.

Note that in order to reduce the level of abstraction and allow for immediate comparability of simulation results to related experimental studies, results are presented with dimensions, except for the film height $h$, which is given in units of the Nusselt flat film thickness

$$
\bar{h}_{N}=\frac{(3 \operatorname{Re})^{1 / 3} v^{2 / 3}}{[g \cos (\alpha)]^{1 / 3}} .
$$

\section{B. Domain size and resolution}

The choice of domain size is crucial for the investigation of spanwise structuring as the domain size is a multiple of the emerging wavelength. Thus, the accuracy of the wavelength to be determined 
(a)

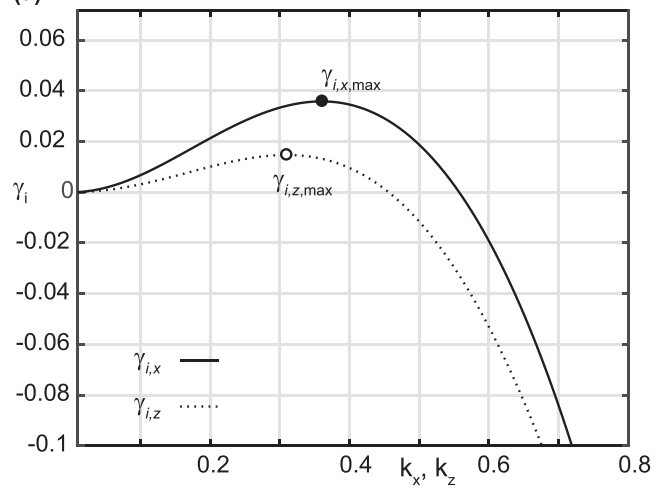

(b)

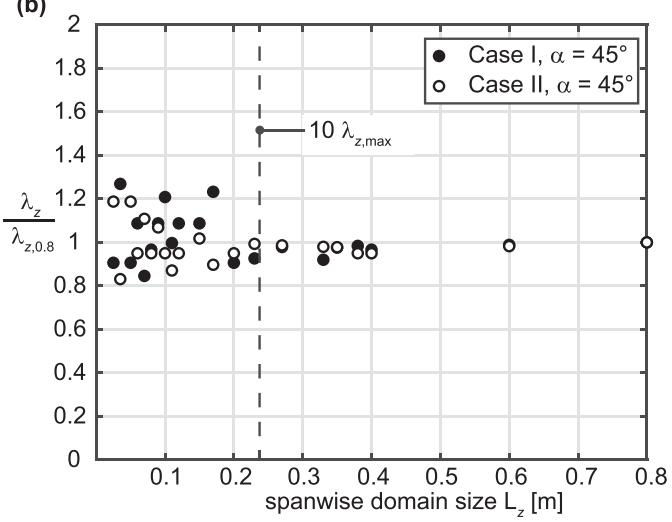

FIG. 2. (a) Linear streamwise and spanwise growth rates $\left(\gamma_{i, x}\right.$ and $\left.\gamma_{i, z}\right)$ of sinusoidal (normal mode) modulations of the film surface with wave number $k_{x}, k_{z}$ for the case $\mathrm{Re}=1 ; \mathrm{Ka}=10 ; \mathrm{Ct}=-1\left(\alpha=45^{\circ}\right)$. (b) Dependence of observed spanwise rivulet wavelengths on spanwise domain size $L_{z}$.

decreases in a smaller domain. In streamwise direction, a domain size containing an uneven number of waves enforces a synchronous pattern formation using periodic boundary conditions. To obtain an estimate of the wavelengths to be expected and thus enable a reasonable choice of domain size, the dispersion relation corresponding to the employed WRIBL model (in 1D form) is utilized (see Appendix A). Note that this expression has been already employed in a previous study [16] and is restated here for completeness.

The most amplified wavelengths in streamwise and spanwise directions can be approximated by the dispersion relation by making use of the nature of the primary instabilities acting on the film. In streamwise direction, the primary instability mechanism can be viewed as a combination of the inertia-driven Kapitza instability and the RT instability induced by destabilizing gravity. In a temporal analysis of Eq. (A3), the growth rate of perturbations with a real wave number $k$ can be obtained by assuming a complex angular frequency $\gamma=\gamma_{r}+\mathrm{i} \gamma_{i}$ and solving Eq. (A3), which is a polynomial in $\gamma$ of degree four, for the root with largest imaginary part. The most amplified wavelength in streamwise direction is the maximum of the obtained dispersion relation $\gamma_{i, x}\left(k_{x}\right)$ [see Fig. 2(a), black continuous line].

For an estimation of the most amplified wavenumber in spanwise direction, negligible impact of inertia is assumed $(\operatorname{Re}=0)$, leading to a simplification of Eq. (A3):

$$
3 i \gamma-3 i k+\left(\frac{27}{5} i \gamma-\mathrm{Ct}\right) k^{2}-\frac{12}{5} i k^{3}-\mathrm{We} k^{4}=0
$$

in which the terms $\frac{27}{5} i \gamma k^{2}$ and $-\frac{12}{5} i k^{3}$ account for viscous dispersive effects [3]. Note that neglecting of those terms in Eq. (10) reduces the dispersion relation to the classical form of the RT type instability for a thin wall bound film [12,13]. Based on experimental observations [11,16], it is assumed that Eq. (10) serves as an appropriate estimate for spanwise wavelengths at least in determining a reasonable domain size. Adding to this, Eq. (10) is used in the remainder of this study as a reference to elucidate in how far the observed evolution of spanwise wavelengths deviates from the classical linear RT mechanism, especially with respect to case II, for which $\operatorname{Re} \gg 1$.

Solving Eq. (10) analogously to Eq. (A3) gives the dispersion relation $\gamma_{i, z}\left(k_{z}\right)=\left.\gamma_{i, x}(k)\right|_{\operatorname{Re}=0}$ for the most amplified wave number in spanwise direction [see Fig. 2(a), black dashed line].

The domain employed in the simulations is constructed based on the determined most amplified linear wave numbers. In the streamwise direction, a multiple of the determined wavelength $\lambda_{x, \max }=\frac{2 \pi \bar{h}_{N}}{k_{x, \text { max }}}$ is chosen. In the spanwise direction, the domain size is chosen so that it is much larger 
than the wavelength of any expected developing rivulets $\lambda_{z \text {, max }}=\frac{2 \pi \bar{h}_{N}}{k_{z, \text { max }}}$. In this way, an influence of the domain size on frequency selection regarding the rivulets is minimized. The influence of spanwise domain size on observed rivulet wavelengths $\lambda_{z}$ is illustrated in Fig. 2(b), for both case I and case II and an inclination of $\alpha=45^{\circ}$. Note that the number of grid points for respective simulations has been selected according to required resolutions as specified in the next paragraph. In Fig. 2(b), determined wavelengths $\lambda_{z}$ are normalized by the wavelength observed for the largest spanwise domain size $\lambda_{z, 0.8}$. In addition, a domain 10 times larger than the most amplified spanwise wavelength corresponding to the displayed cases is indicated [dashed line in Fig. 2(b)] beyond which the value of observed wavelengths converges. The spanwise domain size $L_{z}$ has been chosen to be at least $0.4 \mathrm{~m}$ for all performed simulations.

The spatial resolution of the pseudospectral code WAVEMAKER is determined by the number of modes, fixing in turn the number of grid points by a factor two. Due to the employed method using Fourier transforms, the number of modes is restricted to values equal to a power of two. To identify an appropriate number of grid points, the resolution has been increased in a grid-dependency study from $16 \times 128$ to $128 \times 1024$ grid points for both case I and case II at an inclination angle of $\alpha=45^{\circ}$. A domain in real space of $0.04 \mathrm{~m}$ in streamwise and $0.4 \mathrm{~m}$ in spanwise direction is covered for case I comprising two streamwise wavelengths. For case II, the domain extends over $0.014 \mathrm{~m}$ in streamwise and $0.4 \mathrm{~m}$ in spanwise direction comprising only one streamwise wavelength. For both cases, no convergence was achieved for a resolution lower than $16 \times 128$ grid points.

To evaluate the influence of grid resolution on rivulet formation, the wave topology is shown in Fig. 3 shortly after the spanwise destabilization of 2D waves for each case. Concerning case I, the evolving spanwise wavelength that destabilizes the 2D wave front is similar for all resolutions. However, the number of spanwise waves increases slightly from 15 (lowest resolution) to 16 for the three higher resolutions. In this context, it is mentioned that the spanwise wavelength selection mechanism is not that restrictive as to not allow a noise induced slight variation of the number of evolving waves [see Fig. 2(a)]. Regarding case II, model failure was observed shortly before the destabilization of wavefronts for the two lowest resolutions. Developing wavefronts are seen to be significantly steeper for case II, leading to increased requirements regarding spatial resolution in comparison to case I, for which film height gradients are moderate. A minimal resolution of $64 \times 512$ grid points or higher has been employed for all simulations regarding case I while a minimum resolution of $128 \times 512$ has been employed for case II accounting for the increased film height gradients in the streamwise direction.

\section{Initial conditions}

The initial condition with an initially imposed perturbation on the film surface or flow has a profound influence on the evolution of the film flow, especially on the temporal development. Note that the idea to impose no disturbances and to start with a flat film will also cause an evolution of waves. In this case, the disturbances in the system are caused by numerical rounding errors.

To identify the influence of domain size and the initially imposed disturbance on the film surface, three different initial conditions are employed (see Table II). In this context, $L_{x}$ and $L_{z}$ describe the respective dimensional length and width of the simulation domain. In the streamwise direction, the domain is either twofold (IC1 and IC2) or just onefold of the most amplified wavelength $\lambda_{x \text {, max }}$ (IC3). In the spanwise direction, according to the previous paragraph, a spatial extent of $0.4 \mathrm{~m}$ has been chosen. Note that for low inclinations from the vertical, where large rivulet wavelengths are expected, some simulations have been repeated with an increased value of $L_{z}(0.8 \mathrm{~m})$.

For the initial disturbance, the flat film is superimposed with 2D random noise with a relative amplitude of 0.0001 . We ensure that no mass is added through the initialization of random noise by fixing the first Fourier coefficient of the Fourier transformed film thickness (corresponding to the average film thickness) to the Nusselt flat film thickness. Adding to this, streamwise sinusoidal waves with a relative amplitude of 0.5 are imposed, two waves for IC1 and one wave for IC3. By simulating only one streamwise wavelength in IC3, the influence of wave to wave interactions 


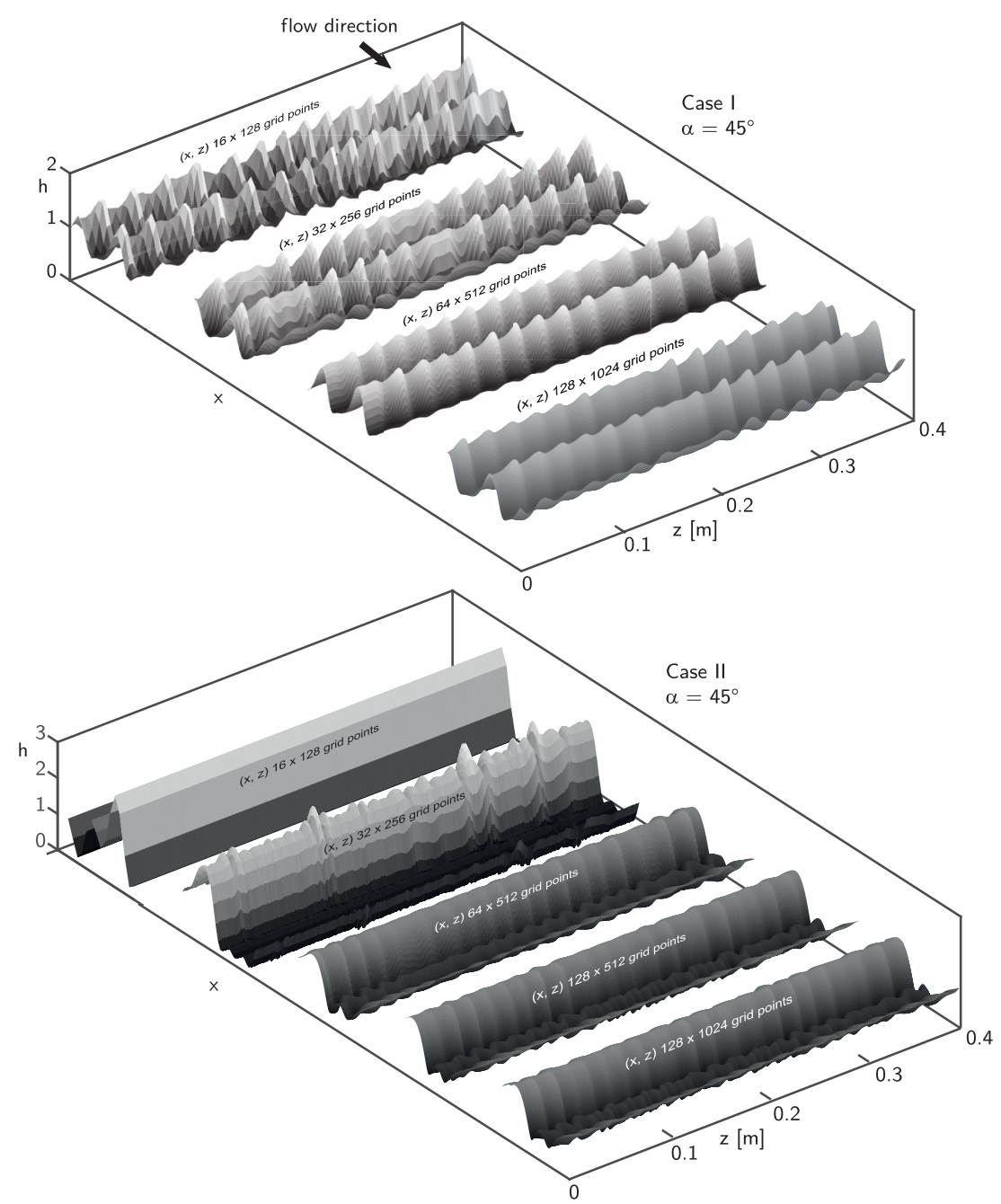

FIG. 3. Determination of required grid resolution. Simulation results are displayed shortly after the spanwise destabilization of $2 \mathrm{D}$ waves or at the instant of model failure: (Top) Exemplary simulation results employing a domain size of $0.4 \mathrm{~m}$ in the spanwise direction and $0.04 \mathrm{~m}$ in the streamwise direction for an increasing number of grid points. Case I: $\mathrm{Re}=1 ; \mathrm{Ka}=13.1 ; \mathrm{Ct}=-1\left(\alpha=45^{\circ}\right)$. (Bottom) Exemplary simulation results employing a domain size of $0.4 \mathrm{~m}$ in the spanwise direction and $0.014 \mathrm{~m}$ in the streamwise direction for an increasing number of grid points. Case II: $\mathrm{Re}=40 ; \mathrm{Ka}=330 ; \mathrm{Ct}=-1\left(\alpha=45^{\circ}\right)$.

on developing rivulets is suppressed. Analogies to the sinusoidal forcing in experimental tests or application are a willingly imposed excitation at the fluid inlet or fluctuations in flow rate due to system components such as pumps and piping. The effect of the amplitude of sinusoidal perturbations on film development has been checked by reducing the amplitude down to a value of 0.2 , using the properties of case I and IC1. In Fig. 4 the evolution of the maximum and minimum film height is presented for two initial amplitudes of sinusoidal excitation, 0.2 and 0.5 , and for two different inclinations. Maximum and minimum film height are shown to reach a constant value after a short time independently of the initial amplitude and depending only on the angle of inclination $\alpha$. In other words, the system chooses its own sustainable amplitude of sinusoidal perturbation as initial condition (depending on $\alpha$ ). Hence, the value of 0.5 has been employed in all simulations. 
TABLE II. Types of initial conditions used in the simulations. The term rand $(x, z)$ describes a random number between zero and one at the position $(x, z)$.

\begin{tabular}{lccc}
\hline \hline & $L_{x}[\mathrm{~m}]$ & $L_{z}[\mathrm{~m}]$ & $h(x, z)$ \\
\hline Initial condition 1 (IC1) & $2 \lambda_{x, \max }$ & $0.4(0.8)$ & $1+0.0001 \operatorname{rand}(x, z)+0.5 \sin \left(\frac{x}{\lambda_{x, \max }} 2 \pi\right)$ \\
Initial condition 2 (IC2) & $2 \lambda_{x, \max }$ & $0.4(0.8)$ & $1+0.0001 \operatorname{rand}(x, z)$ \\
Initial condition 3 (IC3) & $\lambda_{x, \max }$ & $0.4(0.8)$ & $1+0.0001 \operatorname{rand}(x, z)+0.5 \sin \left(\frac{x}{\lambda_{x, \max }} 2 \pi\right)$ \\
\hline \hline
\end{tabular}

\section{RESULTS}

This results section focuses on the film evolution of two distinct flow conditions with fixed values of Reynolds and Kapitza numbers. The first part treats the low Reynolds and Kapitza numbers case (case I). For a comprehensive overview of the film evolution and rivulet formation, exemplary simulation results and observed regimes are presented for IC1-IC3. Subsequently, the growth of spanwise modulations of the film surface is investigated through spectral analysis, which allows us to rationalize influences of initial conditions on spanwise structuring. Finally, the mean spanwise film profile is analyzed in temporal representation, allowing for a quantitative analysis of the evolution of rivulets and rivulet wavelength within the chosen parameter space.

The second part treats a case of intermediate Reynolds and Kapitza numbers (case II) with special attention on differences to case I. Again, spectral analysis as well as representations of the temporal development of the mean spanwise film profile allow for a quantitative analysis of the evolution of spanwise wavelengths.

The third part elucidates observed differences between case I and case II through a parametric study, varying Kapitza number at a constant angle of inclination.

\section{A. Case I: Low Reynolds and Kapitza numbers $\left(\operatorname{Re}=1, \mathrm{Ka}_{\alpha=0^{\circ}}=13.1\right)$}

The temporal evolution of the film flow is illustrated for IC1-IC3 and an exemplary inclination of $\alpha=45^{\circ}$ in Figs. 5 to 7 . In this framework, distinct stages, representative for the variety of

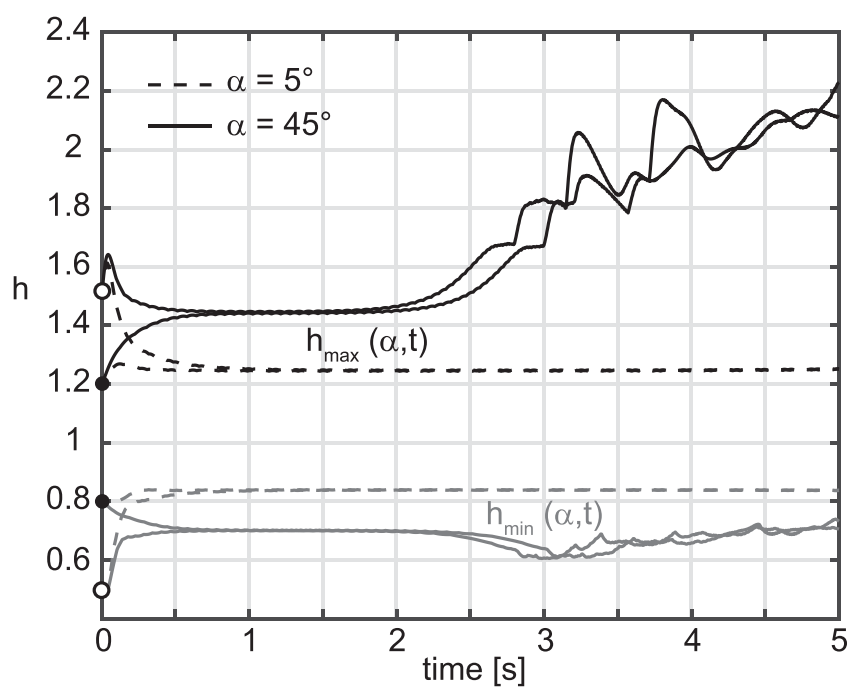

FIG. 4. Influence of the amplitude of initially imposed sinusoidal perturbations on film evolution. The development of the maximum and minimum film height is illustrated for two different initial amplitudes $(0.2$ and 0.5 ) and two different inclinations each, using the properties of case I and IC1. 


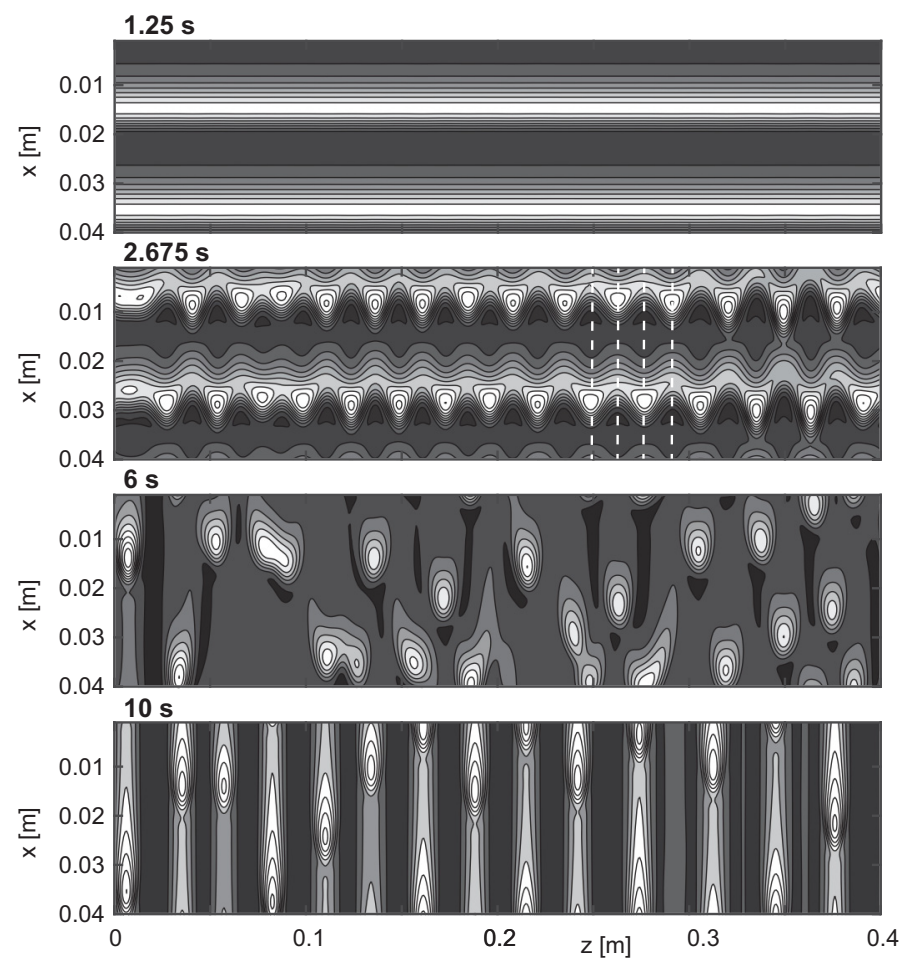

two-dimensional waves

$\mathrm{h}_{\min }=0.70 ; \mathrm{h}_{\max }=1.45$

asynchronous destabilization of 2D waves

$h_{\text {min }}=0.65 ; h_{\text {max }}=1.67$

inception of three-dimensional waves and spanwise coherent structuring

$\mathrm{h}_{\text {min }}=0.64 ; \mathrm{h}_{\max }=2.57$

reorganization into fully developed rivulets

$\mathrm{h}_{\min }=0.17 ; \mathrm{h}_{\max }=4.25$

FIG. 5. Temporal evolution of the film topology for case I exemplarily shown for the case $\mathrm{Re}=1, \mathrm{Ka}=$ 13.1, $\mathrm{Ct}=-1\left(\alpha=45^{\circ}\right)$ employing IC1. Dark colors depict areas of low film height, light colors depict areas of large film height.

observed regimes of film evolution for all investigated inclinations, are displayed. For IC1 (Fig. 5) this includes (1) the formation of 2D waves, (2) their spanwise destabilization, (3) the inception of 3D waves and spanwise structuring, and finally (4) reorganization into fully developed rivulets. Within the first second, distinct 2D waves develop with a steeper front and a more gradual back from the initialized sinusoidal perturbation (see $t=1.25 \mathrm{~s}$ ). These wavefronts destabilize in spanwise direction, in this case in an asynchronous form, i.e., transverse modulations are out of phase for consecutive wavefronts $(t=2.675 \mathrm{~s})$. The asynchronous flow regime as well as the related regime of synchronous destabilization have been observed numerically and experimentally for vertical and positively inclined films. Theoretically the regimes have been predicted by Floquet analysis of 2D traveling wave solutions $[32,33]$. Experimentally both types have been observed, for example, in the comprehensive study of Liu et al. [34] through careful variation of initially imposed noise. It has been shown that the synchronous mode is oftentimes favored in experimental investigations, where spanwise inhomogeneities are generally introduced at the inlet of an experimental setup due to small defects on the blade distributing the film or on the substrate, or due to edge effects. The sensitivity of the transition from asynchronous to synchronous destabilization of consecutive wavefronts with respect to the introduction of additional spanwise noise at initialization is illustrated in Appendix B. The destabilization of $2 \mathrm{D}$ waves is followed by the inception of $3 \mathrm{D}$ waves, accompanied by a breakdown of the highly regular structures (see Fig. 5: time step, 6 s). This regime is characterized by strong interactions between subsequent wavefronts including the merging of waves. In this context, larger and faster wave humps statistically experience more wave-to-wave interactions than smaller and slower ones. This further amplifies the growth of larger waves 


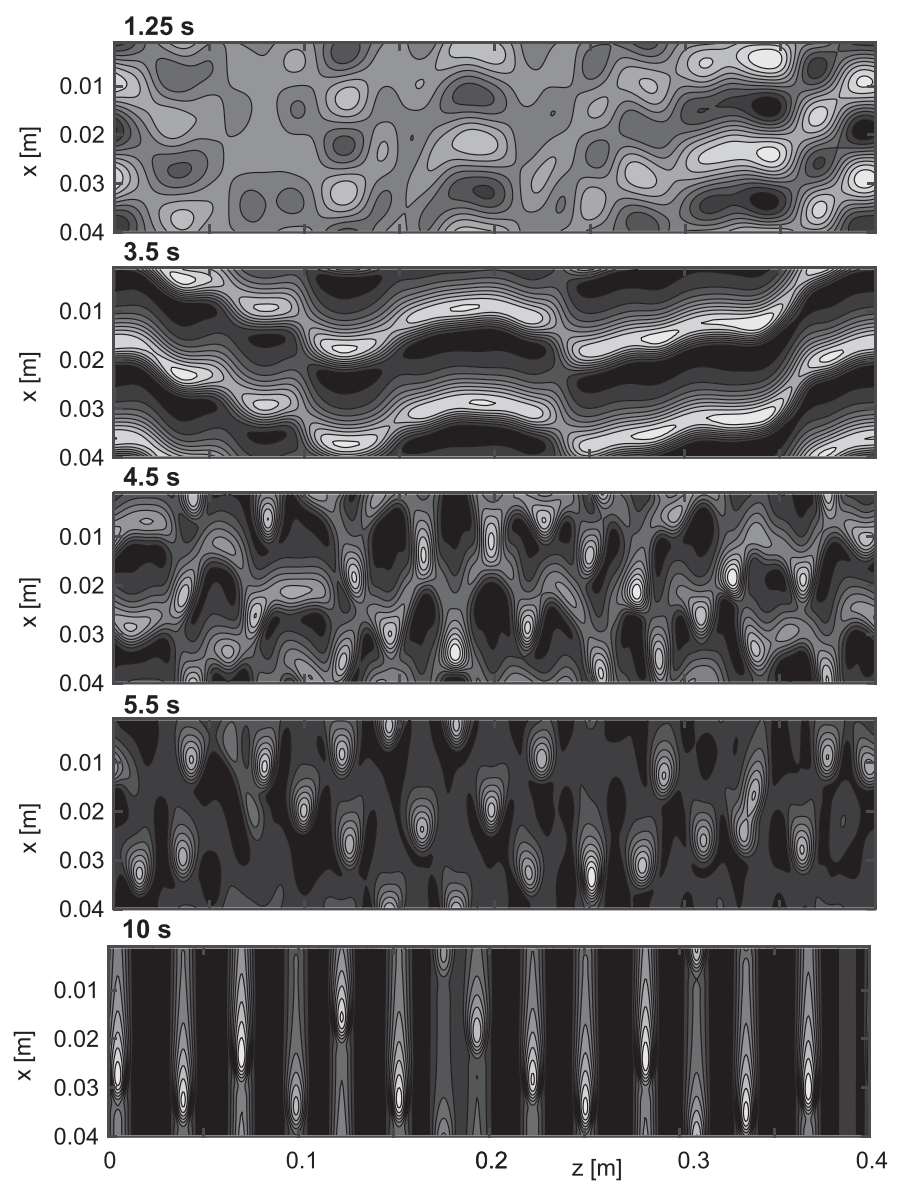

primary growth phase

$h_{\min }=0.9996 ; h_{\max }=1.005$

emergence of modulated continuous wavefronts

$\mathrm{h}_{\min }=0.70 ; \mathrm{h}_{\max }=1.48$

asynchronous destabilization of modulated 2D waves

$h_{\min }=0.63 ; h_{\max }=1.84$

inception of spanwise coherent structuring

$h_{\text {min }}=0.68 ; h_{\max }=2.34$

reorganization into fully developed rivulets

$\mathrm{h}_{\min }=0.14 ; \mathrm{h}_{\max }=4.30$

FIG. 6. Same as Fig. 5 for IC2.

increasing wave height and velocity. Thus, dominant waves act as nuclei for the reorganization into rivulet structures (Fig. 5: time step, $10 \mathrm{~s}$ ).

Significant differences in the early stages of film evolution are observed for IC2, where no streamwise sinusoidal perturbation is initially imposed. For this case, an extended regime of primary growth precedes the emergence of continuous wavefronts (see Fig. $6, t=1.25 \mathrm{~s}$ ). In addition, the developing wavefronts display a weak 3D character (see Fig. 6, $t=3.5 \mathrm{~s}$ ) which has been also commonly observed experimentally for naturally developing waves $[16,35]$. The subsequent film evolution, however, is analogous to IC1 with the asynchronous destabilization of wavefronts, inception of spanwise coherent structuring, and finally reorganization into fully developed rivulets.

The reduced computational domain for IC3 permits only the formation of one wave in the streamwise direction and thereby does not allow for the complex interaction between consecutive wavefronts observed for IC1 and IC2. Consequently, using this particular condition in numerical simulations enforces a specific development and reduces the degrees of freedom. Nevertheless, it offers insights into the film evolution for cases in which no asynchronous destabilization of wavefronts evolves. As a result of the suppression of an asynchronous destabilization of consecutive wavefronts, an extended regime of $3 \mathrm{D}$ waves and reorganization is missing for IC3 (as observed in Fig. 7). In fact, a direct connection between the location of spanwise destabilization of the $2 \mathrm{D}$ wavefront (Fig. 7, $t=3.275 \mathrm{~s}$ ) and spanwise location of emerging rivulets (Fig. 7, $t=9 \mathrm{~s}$ ) is apparent. Despite significant differences regarding the temporal film evolution depending on the 


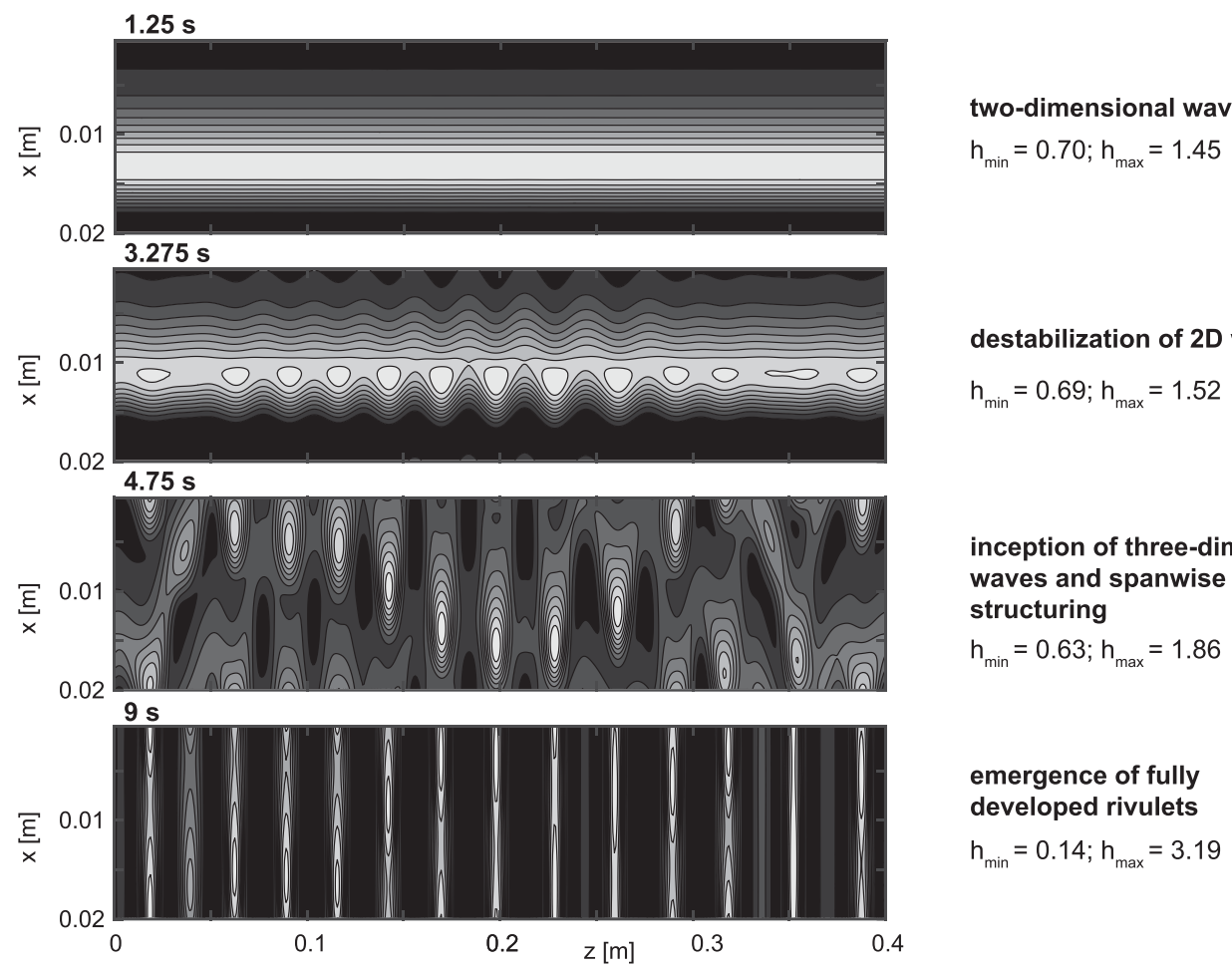

FIG. 7. Same as Fig. 5 for IC3.

chosen initial condition, the final state of fully developed rivulets is almost identical with respect to rivulet wavelength, which suggests that intermediate regimes of asynchronous destabilization and spanwise reorganization are insignificant for the long-term evolution of the film flow. This in turn constitutes evidence for a strong connection between primary instabilities of the film surface and long-term spanwise reorganization into rivulets for case I.

\section{Spectral analysis of spanwise modulations of the film surface}

To quantify the connection between primary instabilities and long-term evolution and especially the influence of initial conditions on primary instabilities, a spectral analysis of spanwise growth rates, most amplified wavelengths, and deformation energies is performed. The analysis is based on the evolution of the mean spanwise film height profile given through

$$
\bar{h}_{\mathrm{z}}(t, z)=\frac{1}{M} \sum_{m=1}^{M} h\left(x_{m}, z, t\right),
$$

where $M$ denotes the number of streamwise grid points (e.g., $M=64)$. Spectral growth rates $\gamma_{i}$ are then obtained through fitting an exponential function to the temporal evolution of respective spatial Fourier coefficients $b(\lambda, t)$ obtained from the Fourier transform of $\bar{h}_{\mathrm{z}}(t, z)$ in the form

$$
b(\lambda, t)=a e^{\gamma_{i}(\lambda) t},
$$

where $\lambda$ is the wavelength of the respective mode and $a$ a constant coefficient. The temporal evolution of spatial Fourier coefficients is exemplarily illustrated in Fig. 8(a) for an inclination of $\alpha=45^{\circ}$ and IC3. For the fit of an exponential function to the data, a short interval of maximally $50 \mathrm{~ms}$ starting at a specific point in time is considered (indicated at $t=1 \mathrm{~s}$ ). One second has been 

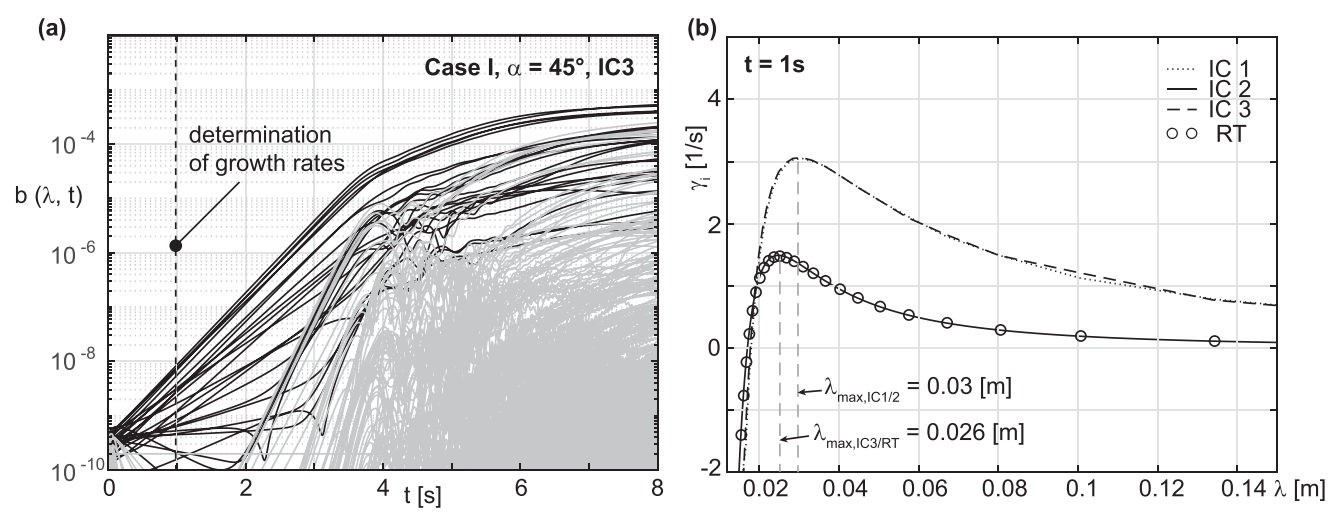

(c) relaxation of

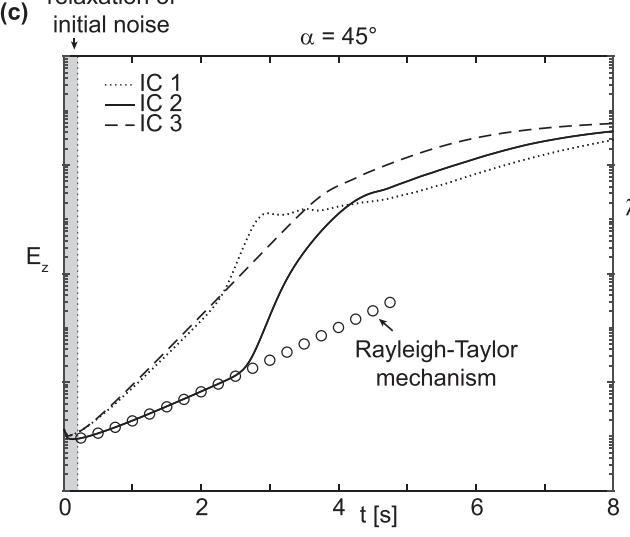

(d)

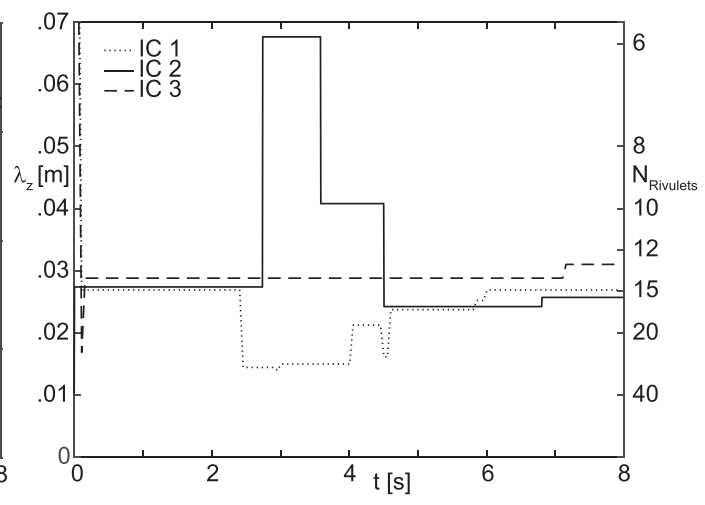

FIG. 8. Spectral analysis of film evolution for case I and $\alpha=45^{\circ}$. (a) Temporal evolution of the spatial Fourier coefficients $b(\lambda, t)$. Coefficients corresponding to dominant wavelengths in the linear growth phase are plotted as black lines. (b) Growth rates at the beginning of film evolution ( $t=1 \mathrm{~s}$ ). Open circles (RT) correspond to growth rates obtained through Eq. (10). (c) Temporal evolution of the spanwise deformation energy $E_{z}$. (d) Temporal evolution of most amplified spanwise wavelengths $\lambda_{z}$.

identified to be small enough for all cases to remain in the linear growth regime, but large enough to have a significant amplitude to be treated. Determined growth rates are disregarded if the signal is not well represented by exponential growth, i.e., $R^{2}<0.95$. Figure $8(\mathrm{~b})$ illustrates the spectrum of growth rates at $t=1 \mathrm{~s}$ for IC1-IC3. Additionally, growth rates determined through Eq. (10) are depicted by open circles. Equation (10) equals the classical formulation of the RT instability, except that viscous dispersive effects are additionally taken into account. An almost exact match between determined growth rates and the RT mechanism is observed for IC2. At the same time, both IC1 and IC3, where the initial condition is characterized through sinusoidal perturbations, show significant deviation from the pure RT mechanism. Note that at $t=1 \mathrm{~s}$ wavefronts with a gradual back and steeper front have already developed from the initial condition for IC1 and IC3. Particularly, increased overall growth rates and a slight increase in the most amplified wavelength are observed, while the critical wavelength (i.e., for $\gamma_{i}=0$ ) remains approximately constant with $\lambda_{c} \approx 0.016 \mathrm{~m}$. It is reasonable to assume that the growth rate spectrum determined for IC1 and IC3 is still associated with a RT-type instability, however modulated through the altered film topology. This is supported by the shape of the spectrum being similar to the one associated with the RT mechanism and the fact that the spectrum is one-peaked, which negates the existence or at least demonstrates the insignificance of additional instability mechanisms acting on the film. Clearly, the 
choice of initial condition significantly impacts the stability of the film flow in the linear stage of development.

How the linear growth phase transitions into the late stages of film evolution is well illustrated through the development of spanwise deformation energies as depicted in Fig. 8(c). The spanwise deformation energy $E_{z}(t)$ is defined here as the quadratic sum of the spatial Fourier coefficients obtained from the Fourier transform of $\bar{h}_{\mathrm{z}}(t, z)$ as

$$
E_{z}(t)=\frac{1}{N}\left(\sum_{n=1}^{N} b\left(\lambda_{n}, t\right)^{2}\right)^{1 / 2}
$$

The temporal development of $E_{z}$ is depicted in Fig. 8(c) in semilogarithmic representation again for IC1-IC3 and for the RT mechanism included in the dispersion relation (10), which was determined by assuming the corresponding growth rate spectrum acting on the film being independent of film topology and hence independent of time. This allows to identify the point in time for which a pure RT mechanism fails to describe the evolution of spanwise structuring for IC2, namely, at approximately $t=2.7 \mathrm{~s}$, which corresponds to emergence of significant 2D waves on the film surface and long wave modulations of the latter (see Fig. 6). An abrupt change in slope is also seen for IC1 corresponding to the asynchronous destabilization of consecutive wavefronts at approximately $t=2.4 \mathrm{~s}$. Meanwhile, IC3 displays no abrupt changes in slope and directly transitions into a regime of saturation. Interestingly, the initial film evolution is characterized through a distinct phase of primary growth for all ICs, with IC1, and IC3, however, displaying larger slopes of the respective graphs corresponding to increased growth rates. How the described evolution of deformation energy is related to the temporal evolution of most amplified spanwise wavelengths is illustrated in Fig. 8(d). Clearly, most amplified wavelengths in the early stage of film evolution are very similar to most amplified wavelengths in the late stages of film evolution, indicating a connection between primary growth and long-term spanwise structuring. The intermediate most amplified modes of short and long wavelengths for IC1 and IC2, namely, asynchronously destabilizing consecutive wavefronts and modulated 2D wavefronts, do not remain dominant for the late stages.

\section{Temporal evolution of the mean spanwise film height profile}

To further address the influence of primary instabilities on long-term spanwise structuring, a parametric analysis has been performed varying both wall inclination and initial condition. From this perspective, film evolution within the parameter space is investigated through temporal representations of the mean spanwise film topology $\bar{h}_{z}(t, z)$, Eq. (11). To visualize the temporal film evolution from the beginning, where very small spanwise variations are present, to the late stages, where large amplitude quasistationary rivulets are formed, the mean spanwise film topology is normalized based on the minimum and maximum values at each respective time step through

$$
\bar{h}_{z, \text { norm }}(t, z)=\frac{\bar{h}_{\mathrm{z}}(t, z)-\bar{h}_{z, \min }(t)}{\bar{h}_{\mathrm{z}, \max }(t)-\bar{h}_{\mathrm{z}, \min }(t)} \quad \text { with } \quad \bar{h}_{z, \operatorname{norm}}(t) \in[0,1] .
$$

The results are visualized in Figs. 9 to 11 for inclination angles $0^{\circ}, 5^{\circ}, 15^{\circ}, 30^{\circ}, 45^{\circ}$, and $75^{\circ}$ and initial conditions IC1, IC2, and IC3. For orientation, the end of the primary growth phase of spanwise modulations $t_{\mathrm{P} \text {, end }}$ is indicated for IC1 and IC2, $t_{\mathrm{P} \text {, end }}$ being characterized according to Fig. 8(c) through an abrupt change in slope of the corresponding evolution of spanwise deformation energy.

The physical simulation time varies between the different cases and decreases systematically with increasing inclination. For an inclination angle of $\alpha=0^{\circ}$ (vertical plate), the film flow evolves over more than $100 \mathrm{~s}$ owing to low growth rates of hydrodynamic instabilities. For high inclinations $\left(\alpha=75^{\circ}\right)$ not more than $2.6 \mathrm{~s}$ of film evolution are shown. Note that simulation results for $\alpha=45^{\circ}$ and $\alpha=75^{\circ}$ are terminated at the point in time when a very high (almost infinite) slope of the film topology occurs within the simulation domain. In this case, the employed modeling technique fails. 

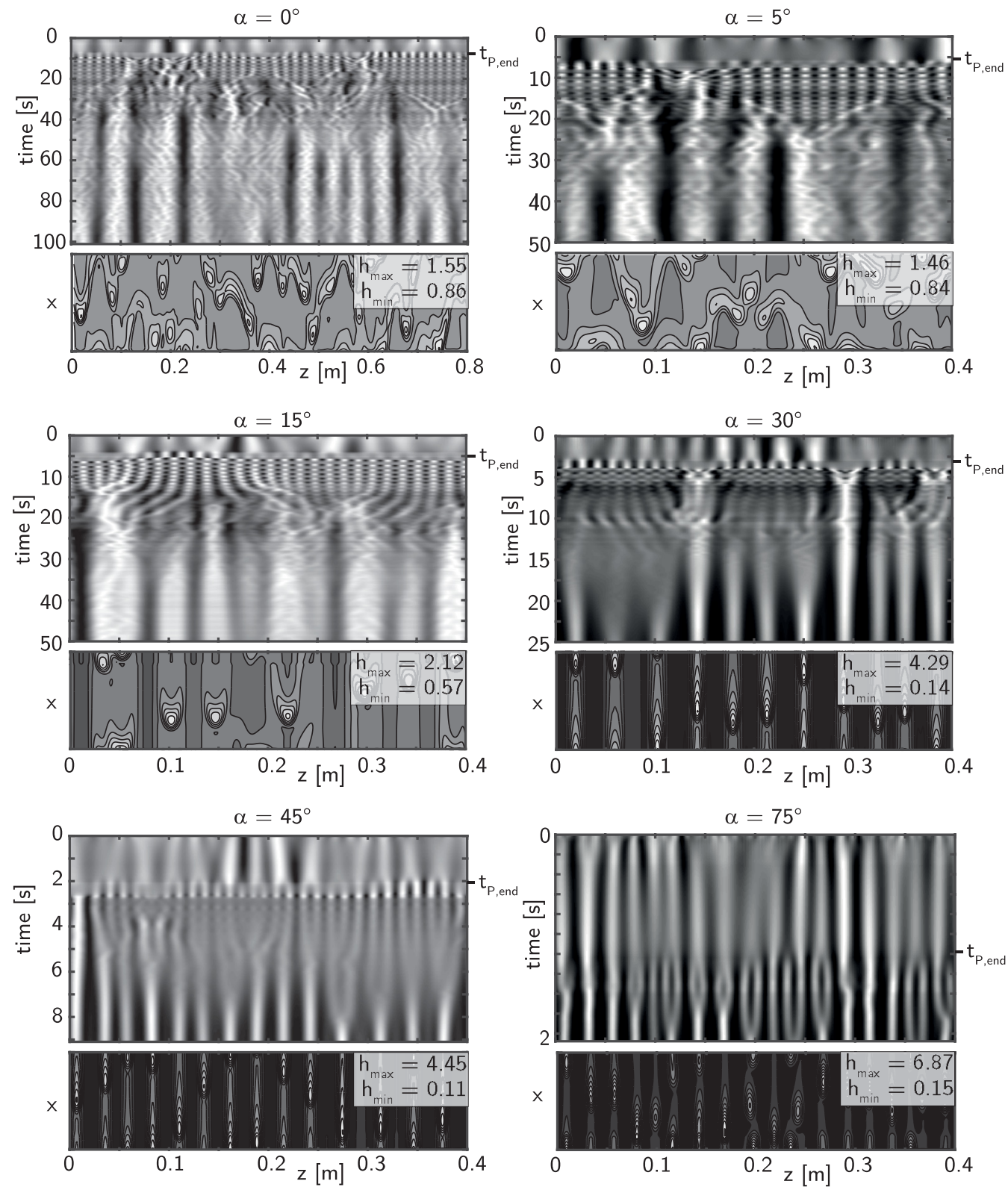

FIG. 9. Temporal plots of the average spanwise film height profile $\bar{h}_{z, \text { norm }}(t, z)$ and spatial plots of the last simulated time step using IC1. The temporal development is illustrated for various inclination angles. Dark to light colors span the value range [0,1] of the normalized mean spanwise film topology (see Eq. (14)). Spatial plots of the last time step are normalized by the minimum and maximum film height. 

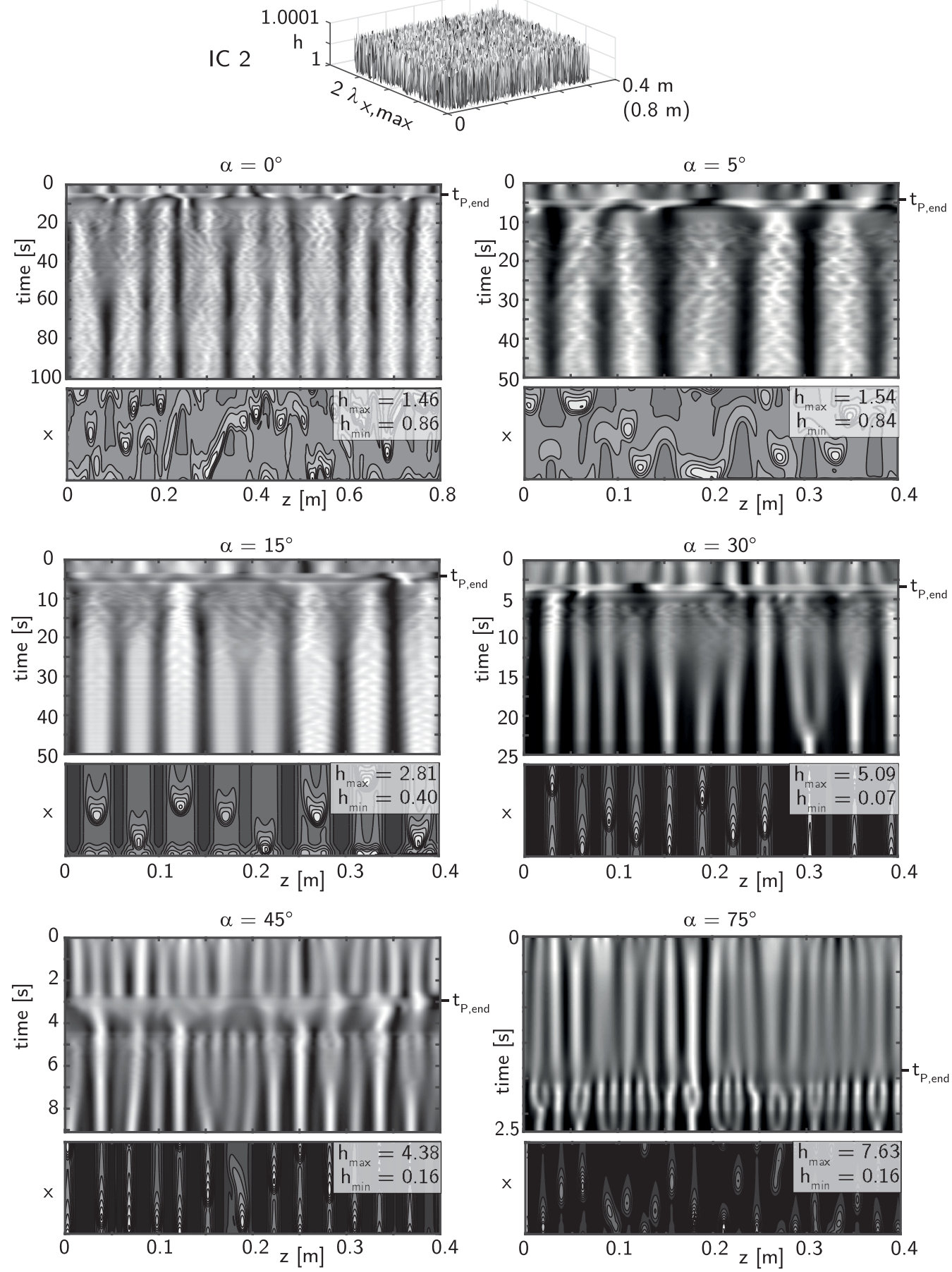

FIG. 10. Same as Fig. 9 for IC2. 
IC 3
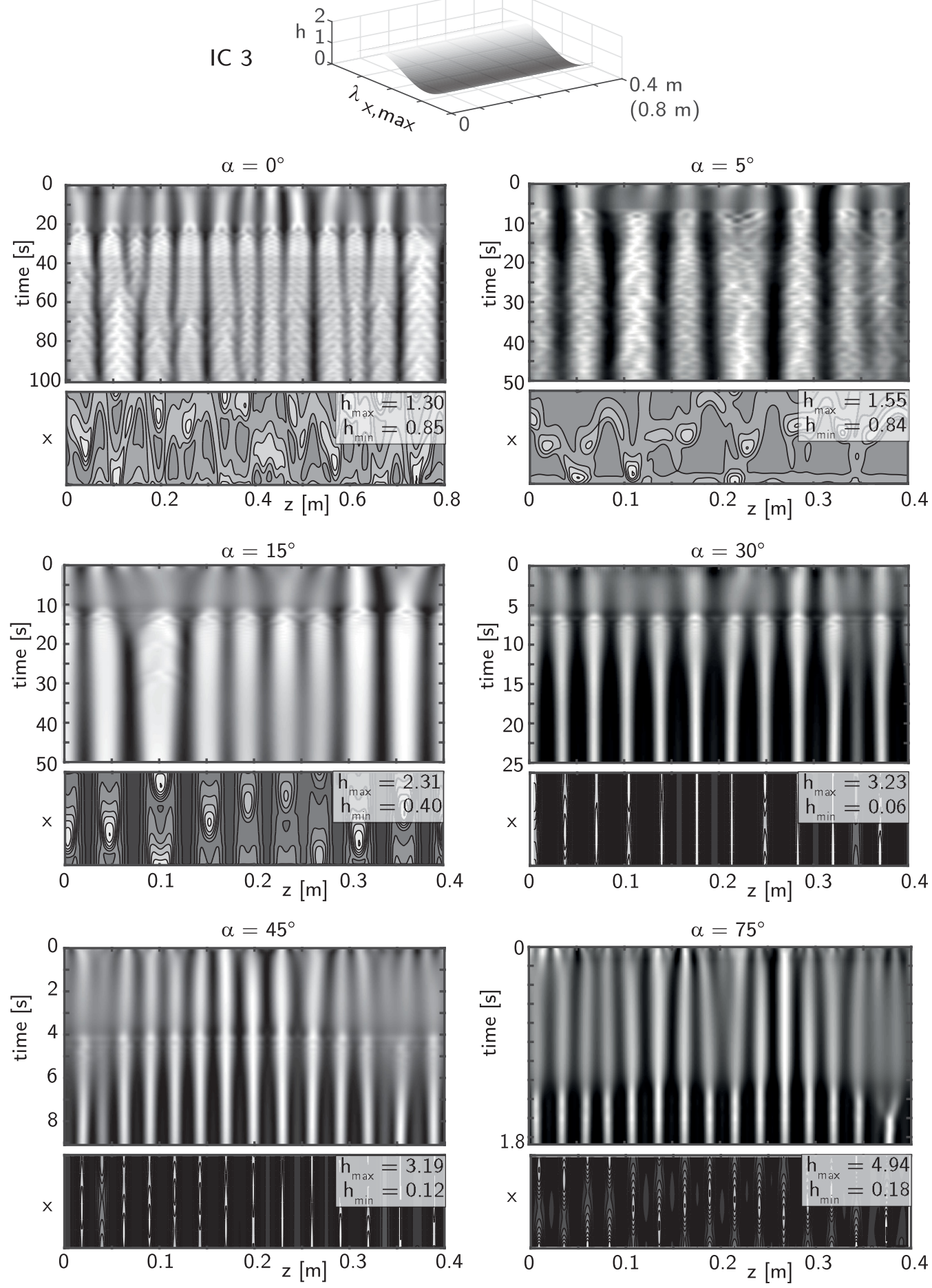

FIG. 11. Same as Fig. 9 for IC3. 


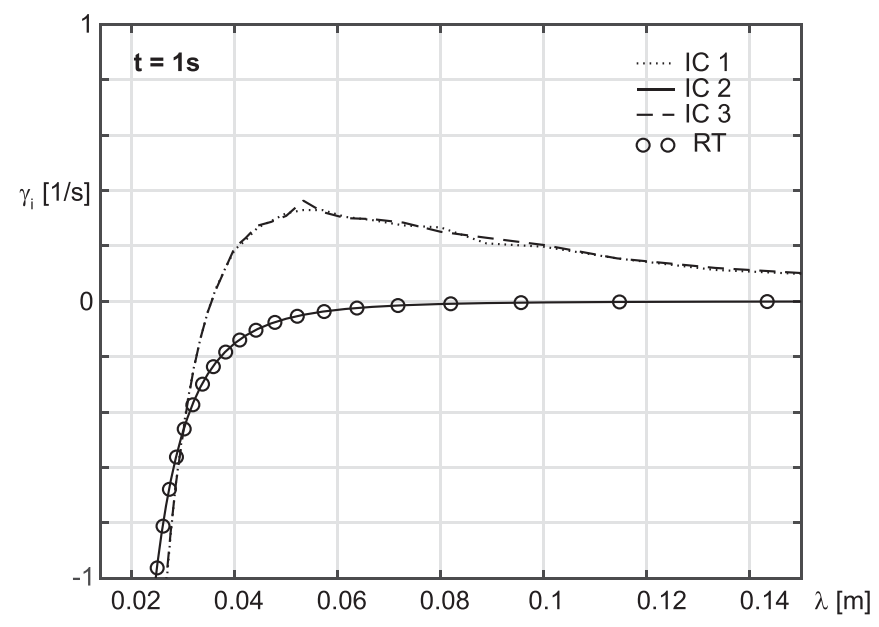

FIG. 12. Spanwise growth rates at the beginning of film evolution $(t=1 \mathrm{~s})$ for $\alpha=0^{\circ}$. Open circles (RT) correspond to growth rates obtained through Eq. (10).

The strong steepening of the wave can be associated with the formation of droplets and hence to the beginning of dripping [36]. Below each temporal evolution, the spatial film topology is shown for the last simulated time step.

For all cases including a vertical wall $\left(\mathrm{Ct}=0 ; \alpha=0^{\circ}\right)$ spanwise redistribution of fluid is observed (see Figs. 9 to 11), which leads to a spanwise modulation with alternating locations of increased and respectively decreased average film height. At the same time, the final spatial film topology reveals a significantly different flow behavior between the cases of suspended films $\left(\alpha>0^{\circ}\right)$ and the vertical wall case, i.e., $\alpha=0^{\circ}$. The destabilizing gravitational force normal to the wall for $\alpha>0^{\circ}$ leads to the formation of distinct rivulets separated by thin film areas. Note that rivulet formation is only weakly observable for small inclinations from the vertical $\alpha=5^{\circ}$. Rivulet width, or equivalently rivulet wavelength, is shown to decrease with increasing inclination from the vertical in agreement with recent experimental studies [11,16,25]. For $\alpha=0^{\circ}$, i.e., without the destabilizing action of gravity, temporal plots may also suggest the existence of rivulets. However, the spatial film topology displays no distinct rivulet development at $t=100 \mathrm{~s}$. Rather, spanwise coherent structuring observed in the temporal evolution of the average spanwise film height profile might be seen as the trace of dominating wavefronts formed earlier in the process of film evolution connected to the destabilization of 2D wavefronts. Kofman et al. [37] have demonstrated that gravity can still destabilize 2D wavefronts in the sense of an instability mechanism of inertial origin connected to the curvature of the wavefront for $\alpha \leqslant 0^{\circ}$. This is confirmed for case I by Fig. 12, illustrating the growth rate spectrum for $\alpha=0^{\circ}$ and IC1-IC3. For IC2 no growth of spanwise modulations $\left(\gamma_{i}<0\right)$ is present at $t=1 \mathrm{~s}$ as predicted from linear stability analysis of the initially flat film. At the same time, both IC1 and IC3 lead to a single peaked spectrum with positive growth rates over a significant range of spanwise wavelengths. The same mechanism is supposed to account for rivulet emergence as observed by Kharlamov et al. [35] for a vertical falling liquid film.

The imposed initial condition is shown to significantly influence the temporal evolution of the spanwise redistribution process of the fluid (see Figs. 9 and 11). This can have important implications for experimental and numerical studies. First, the asynchronous destabilization of consecutive 2D waves, most pronounced in IC1, leads to an extended region of decreased spanwise wavelengths (see case $\mathrm{IC} 1,15^{\circ}$, about $6 \mathrm{~s}$ to $14 \mathrm{~s}$ after initialization) and a subsequent pronounced region of spanwise reorganization (see case $\mathrm{IC} 1,15^{\circ}$, about $14 \mathrm{~s}$ to $30 \mathrm{~s}$ after initialization). These regions shrink and move closer to film initialization $(t=0 \mathrm{~s})$ for an increasing inclination from the vertical. This is grounded both in the increase of the growth rate of the primary hydrodynamic 

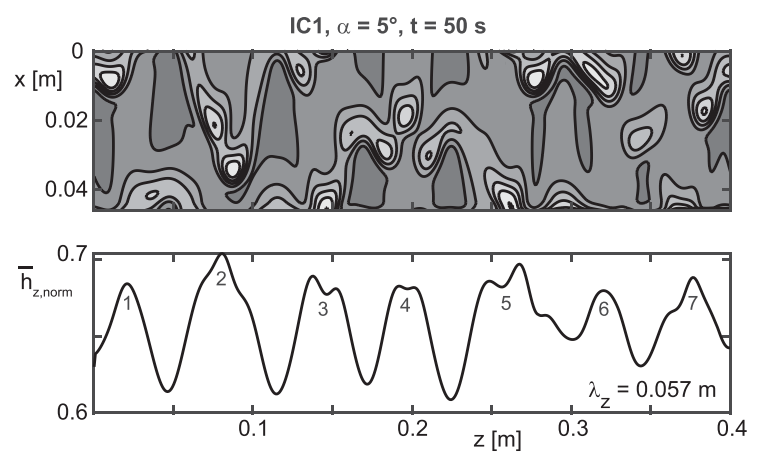

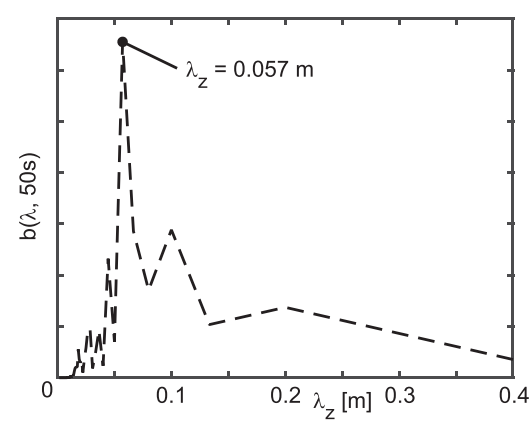

FIG. 13. Determination of the dominant spanwise wavelength, illustrated for $\alpha=5^{\circ}$ and IC1. Left: Determination of the final rivulet wavelength by manually counting the number of spanwise rivulets. Right: Determination of the dominant final rivulet wavelength though spectral analysis.

instability, which supports the growth of $2 \mathrm{D}$ waves, as well as that of the RT instability. The latter drives the reorganization of the 3D wave regime into separate rivulets. As a consequence, experimental evaluation of the spanwise film topology at a fixed position and for an increasing inclination might lead to the observation of a regime change with respect to rivulet wavelengths, which is in fact a decreasing inception length of the above described regimes. Less pronounced regimes of locally decreased spanwise wavelength are visible for IC2, for example, for an inclination of $15^{\circ}$ around the $5 \mathrm{~s}$ mark and for an inclination of $75^{\circ}$ around the $2.5 \mathrm{~s}$ mark. A cause of this difference to IC1 can be found in the initially developing wavefronts. In contrast to IC1, these are not $2 \mathrm{D}$, but highly $3 \mathrm{D}$ while still forming a connected wave. In fact, in Fig. 10, the moment of formation of connected 3D wavefronts is clearly visible through an abrupt change of the mean spanwise wave topology profile (IC2: $0^{\circ}$, at about $8 \mathrm{~s} ; 15^{\circ}$, at about $5 \mathrm{~s} ; 45^{\circ}$, at about $3-4 \mathrm{~s}$ ). The $3 \mathrm{D}$ character of these wavefronts hinders the development of structures with high symmetry therefore promoting wave interactions and reorganization into rivulets. For IC3, the connection between spanwise wavelength selection in the linear growth phase and long-term spanwise structuring is apparent for all investigated inclinations.

\section{Wavelength of spanwise structures}

In the following, a quantification of observed spanwise wavelengths within the investigated parameter space is conducted to further elucidate the connection between initial growth rates of spanwise perturbations and long-term spanwise structuring. For this purpose, dominant spanwise wavelengths at the end of film evolution are compared to the initially most amplified spanwise wavelength determined from the growth rate spectrum of the average spanwise wave-height profile [see Figs. 8(b) and 12] and the primary RT mechanism.

In this context, dominant spanwise wavelengths at the end of film evolution are determined by dividing the domain size in spanwise direction by the number of manually counted rivulets (see Fig. 13, left) and additionally through spectral analysis of the average film height profile (see Fig. 13, right). Precision is given for the wavelengths determined through counting of rivulets considering the error which would occur if the number of rivulets would be increased or respectively decreased by one. The results are illustrated in Fig. 14 for IC1-IC3, together with the most amplified wavelength of the primary RT instability (continuous line) as a function of the inclination angle,

$$
\lambda_{R T}=2 \pi \sqrt{2} l_{c} \frac{1}{\sqrt{\sin \alpha}},
$$

as well as the most amplified wavelength determined from the growth rate spectrum of spanwise perturbations at $t=1 \mathrm{~s}$, i.e., within the linear growth phase (dashed line). Note that Eq. (15) directly 

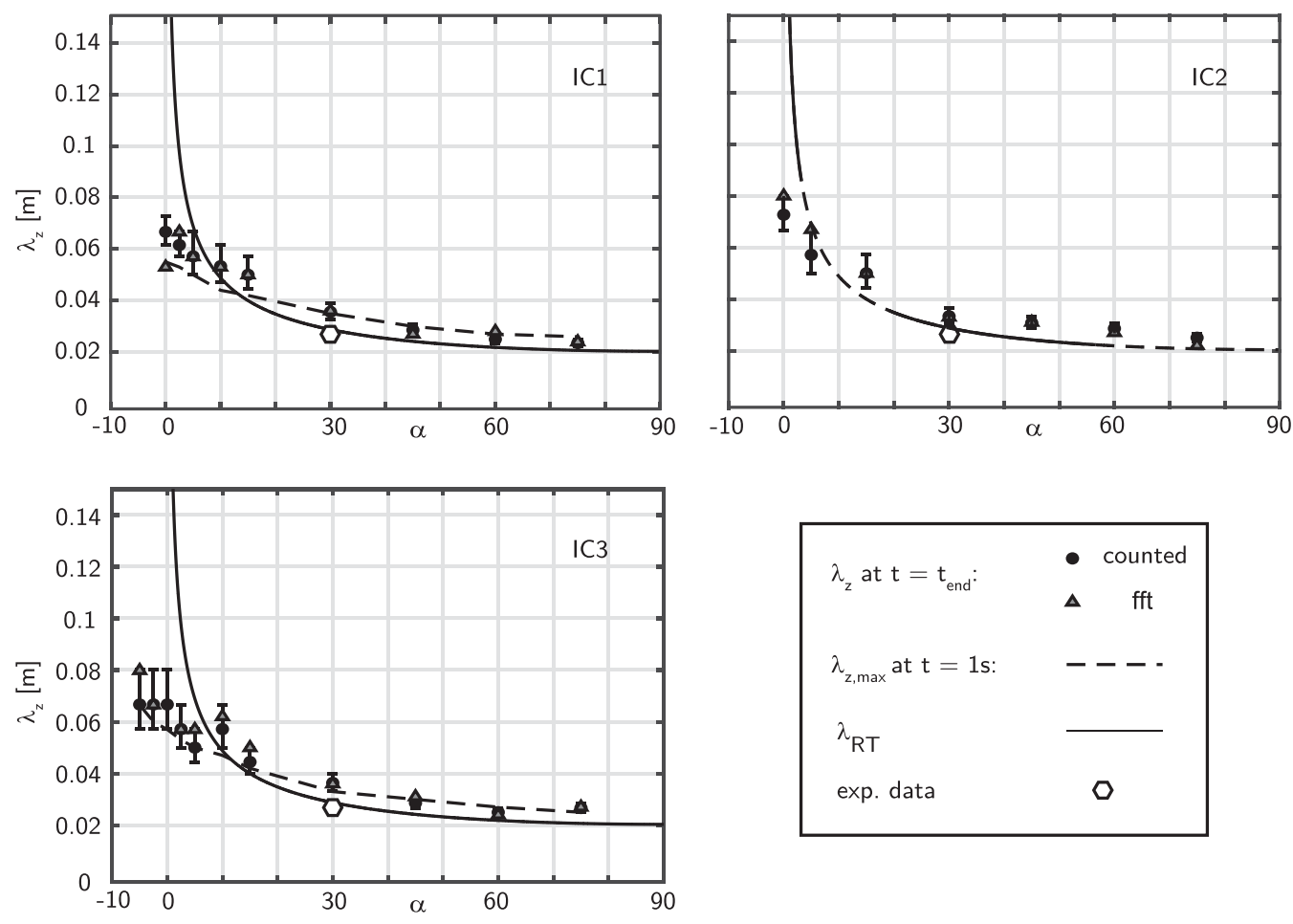

FIG. 14. Quantitative evaluation of the wavelength of spanwise structuring (rivulet formation) for IC1-IC3 under variation of wall inclination. Most amplified wavelength of the primary RT instability $\lambda_{\text {RT }}$ (continuous line) as a function of the inclination angle and the most amplified wavelength $\lambda_{z \text {,max }}$ determined from the growth rate spectrum of spanwise perturbations at $t=1 \mathrm{~s}$, i.e., within the linear growth phase (dashed line), are indicated. Experimental data points taken from [11].

follows from Eq. (10) under neglecting viscous dispersive effects. Within the investigated parameter space, Eqs. (15) and (10) give almost exactly the same result for the most amplified spanwise wavelength. Therefore, only Eq. (15) is plotted. Additionally, accessible experimental data from the recent study of Charogiannis et al. [11] are indicated. Exact initial conditions of the respective experiment are unknown. Therefore, the data point is added to the plot of all three different ICs employed within this study.

Most notably, Fig. 14 illustrates that final wavelengths of spanwise structuring are strongly correlated to the most amplified wavelengths determined from the growth rate spectrum of spanwise perturbations within the linear growth phase (dashed line). Predominantly for IC1 and IC3, it is apparent that this remains true for small angles from the vertical and even angles where $\alpha<0^{\circ}$, as illustrated for IC 3 . Note that in the case of IC2 these wavelengths coincide with $\lambda_{\mathrm{RT}}$, while, as has been shown earlier (see Fig. 8), for IC1 and IC3 growth rates differ from $\lambda_{\mathrm{RT}}$ due to initialized sinusoidal perturbations. Meanwhile, $\lambda_{\mathrm{RT}}$ fails to predict spanwise structuring for small inclinations from the vertical, where corresponding growth rates are low, and is seen to serve as a good estimator for final rivulet wavelengths only for $\alpha>5^{\circ}$. In other words, for small inclinations from the vertical spanwise structuring is determined through an instability mechanism connected to the developing wavefronts (secondary instability) more than through the primary RT instability. A reasonable match between experimental data [11] and performed simulations is observed, even if experimental data for a validation of the generally observed trend is missing. 


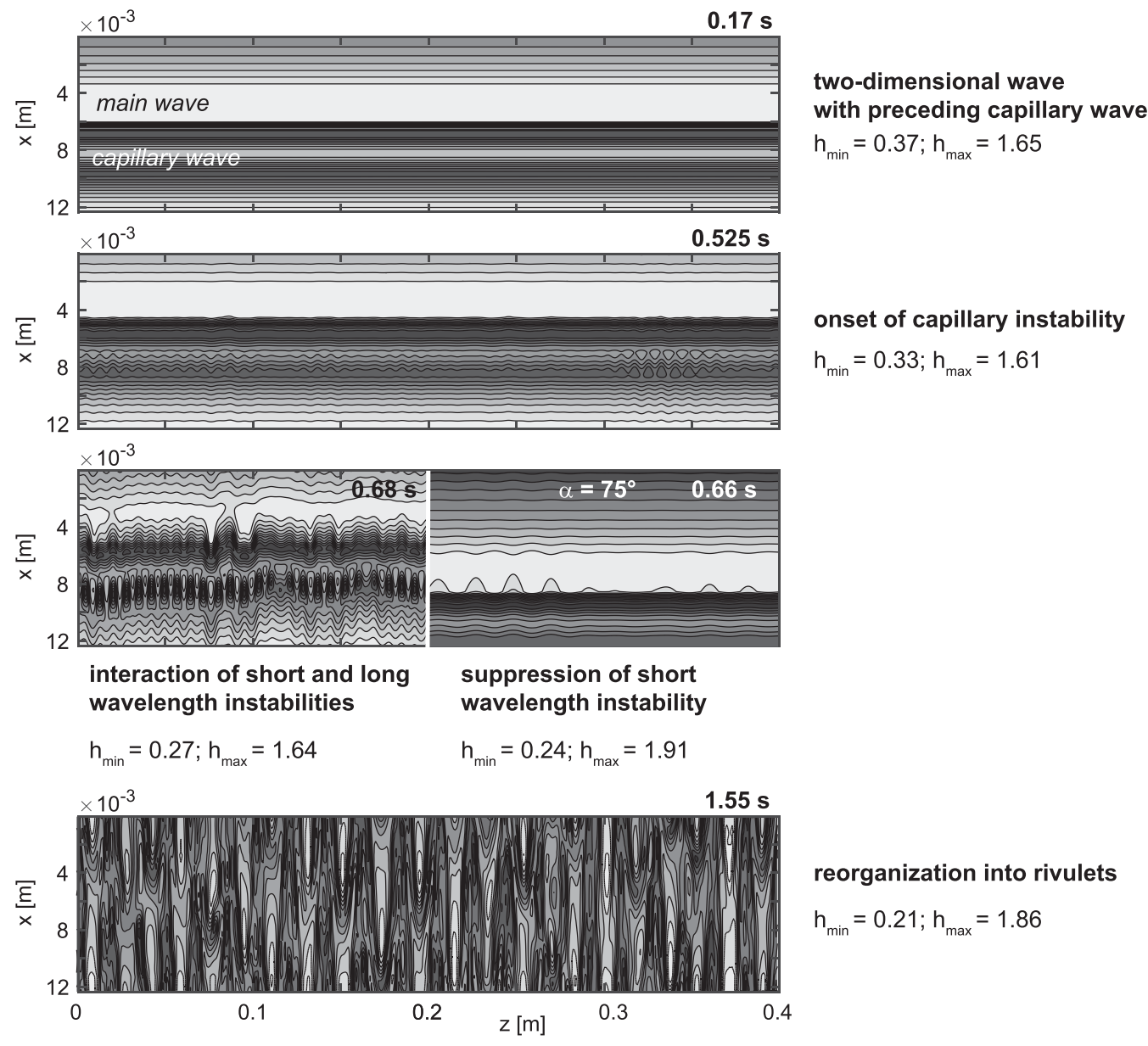

FIG. 15. Temporal evolution of the film topology for case II exemplarily shown for the case $\mathrm{Re}=40 ; \mathrm{Ka}=$ 330; $\mathrm{Ct}=-0.58\left(\alpha=30^{\circ}\right)$ employing IC3. Additionally, the suppression of a short wavelength instability is illustrated for $\alpha=75^{\circ}(\mathrm{Ct}=3.73)$. Dark colors depict areas of low film height, light colors depict areas of high film height.

\section{B. Case II: Increased Reynolds and Kapitza numbers $\left(\operatorname{Re}=40, K_{\alpha=0^{\circ}}=330\right)$}

The flow conditions of case II are characterized by a stronger influence of inertial forces (higher Reynolds number) and a stronger influence of capillary forces (higher Kapitza number). As such, growth rates of initial disturbances are larger, and observed wave patterns are of much higher complexity. Owing to the increase in computational effort, simulations of case II have been performed only utilizing IC3, which hinders only the discussion of synchronous/asynchronous destabilization of consecutive wavefronts.

Figure 15 illustrates the temporal evolution of the film topology for $\alpha=30^{\circ}(\mathrm{Ct}=-0.58)$. Note that the timespan in which the different stages of film evolution occur is significantly shortened in comparison to case I because of the increased growth rates of fluid dynamical instabilities. For the same reason, simulation failure due to potential dripping events occurs much earlier, hindering simulations deep into the regime of rivulet formation, especially for large inclinations from the vertical.

Differences to case I are already observed in the development stage of quasi-2D waves which emerge from the initialized sinusoidal perturbation. The stronger influence of surface tension causes 
a pronounced capillary wave pattern that precedes the main wave hump (visualized in Fig. 15 at $t=0.17 \mathrm{~s}$ ). The capillary wave pattern is subject to a short wavelength instability that originates in the capillary wave region and invades the remainder of the fluid domain $(0.525 \mathrm{~s})$. In a later stage (at about $t=0.68 \mathrm{~s}$ ) the main wave hump (located at $\approx 3 \mathrm{~mm}$ ) begins to destabilize, however with a much larger wavelength compared to the one of the capillary waves. Within a regime of strong interactions between short- and long-wave instabilities of the capillary waves and the main wave hump, respectively, the flow reorganizes into rivulet structures.

Note that the emergence of a short wavelength instability with capillary origin has not been observed for $\alpha>45^{\circ}$ in case II. This is exemplarily shown in Fig. 15 for $\alpha=75^{\circ}$. In this case, the main wave hump destabilizes with a distinct wavelength which is imprinted onto the whole fluid domain including the preceding capillary wave, which shows no destabilization through an instability with short wavelength. Hence, the long wavelength instability of the main wave hump dominates the process of spanwise structuring of the film topology for sufficiently large inclination angle.

\section{Spectral analysis of spanwise modulations of the film surface}

Analogously to case I, a spectral analysis of spanwise growth rates, most amplified wavelengths and deformation energies is performed in the following. Due to the regime change regarding the dominance of a short wavelength instability of capillary waves or a long wavelength instability of the main wave hump during the early film evolution (see Fig. 15), the analysis is carried out for two inclinations, $\alpha=30^{\circ}$ and $\alpha=60^{\circ}$.

Figure 16(a) depicts the wave profile for both inclinations before a significant spanwise destabilization is observed. Both waves are characterized by a distinct capillary wave preceding the main wave hump.

Corresponding spectra of growth rates of spanwise wavelengths are illustrated in Fig. 16(b). Matching the observation of the existence of long and short wavelength instabilities, two peaks are found for both inclinations. For $\alpha=30^{\circ}$, growth rates of the short-wave mode $\left(\lambda_{\text {short-wave }} \approx\right.$ $0.008 \mathrm{~m}$ ) exceed growth rates of the long-wave mode $\left(\lambda_{\text {long-wave }} \approx 0.02 \mathrm{~m}\right)$. This leads to the observed dominance of a short wavelength instability of the capillary wave observed in Fig. 15. For $\alpha=60^{\circ}$ the long-wave mode dominates, while the short-wave instability is suppressed. Meanwhile, both spectra differ remarkably from respective spectra associated with the RT instability, Eq. (10). Most notably, growth rates of the long wavelength instability are seen to approximately remain comparable for both inclinations, while the RT mechanism predicts a significant increase according to the increasing angle from the vertical. Additionally, the most amplified long-wave mode differs significantly from the one associated with the primary RT mechanism for $\alpha=30^{\circ}$.

The temporal evolution of spanwise deformation energy $E_{z}$ in Fig. 16(c) displays a phase of constant growth following the relaxation of initially imposed noise, which directly transitions into a regime of saturation. This is comparable to the evolution of spanwise deformation energy observed for IC3 regarding case I. Note that the regime of saturation could not be resolved for $\alpha=60^{\circ}$ due to model failure, which points to the emergence of an infinite slope within the domain at $t \approx 0.8 \mathrm{~s}$.

Regarding the temporal evolution of dominant spanwise wavelengths [Fig. 16(d)], for $\alpha=30^{\circ}$, observed values are confined by the short-wave and the long-wave mode. An intermediate value of $\lambda_{z} \approx 0.017 \mathrm{~m}$ is reached at the end of the simulation. For $\alpha=60^{\circ}$, after a short initial growth phase, the dominant spanwise wavelength is seen to correspond to the long-wave mode. Note that the corresponding wavelength $\lambda_{z}=0.025 \mathrm{~m}$ is close to the most amplified wavelength associated with the RT mechanism, as obtained through either Eq. (10) or (15).

\section{Temporal evolution of the mean spanwise film height profile}

For case II, the film evolution within the investigated range of inclinations is analyzed analogously to case I through temporal representations of the mean spanwise film topology $\bar{h}_{\mathrm{z}}(t, z)$ 


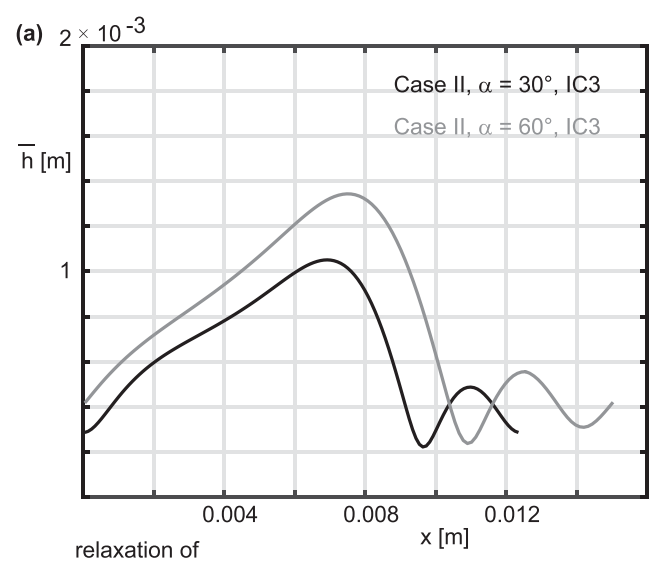

(c) initial noise
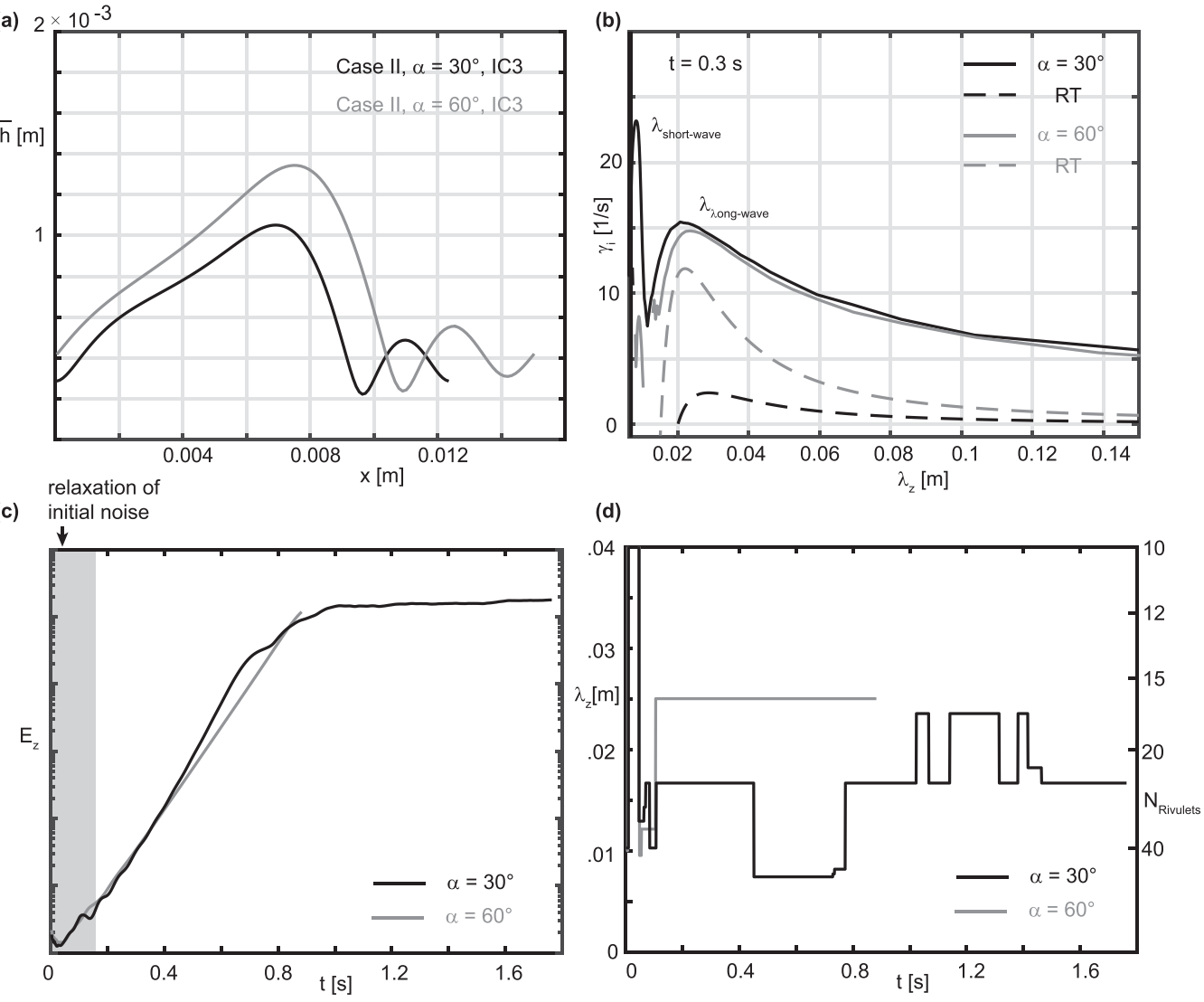

FIG. 16. Spectral analysis of film evolution for case II and for inclination angles $\alpha=30^{\circ}$ and $\alpha=60^{\circ}$. (a) Profile of initially developing waves; (b) growth rates at the beginning of film evolution $(t=0.3 \mathrm{~s})$. Dashed lines (RT) correspond to growth rates obtained through Eq. (10) for the respective inclinations. (c) Temporal evolution of spanwise deformation energy $E_{z}$. (d) Temporal evolution of most amplified spanwise wavelengths $\lambda_{z}$.

(Eq. (11)) in Fig. 17. For the case of a vertical wall $\left(\alpha=0^{\circ}\right)$, only very weak spanwise reorganization is observed within the simulated timespan. While rivulet-like structures are recognizable in the temporal plot of the average spanwise film height, no alignment parallel to the flow direction is seen, indicating an extended regime of wave interaction. In accordance with results presented in Fig. 16, for low inclinations from the vertical, strong interactions between short- and long-wave instabilities are seen to significantly influence spanwise structuring and rivulet formation. Particularly, the early dominance of the short-wave mode is visible as a high-frequency modulation of the mean spanwise film height profile for inclination angles $\alpha=0^{\circ}-30^{\circ}$. With an increasing angle from the vertical, alignment of rivulet structures with the flow direction rapidly increases, pointing to an increasing influence of the RT instability. Meanwhile, temporal changes of dominant spanwise wavelengths are clearly observable. Exemplarily, for $\alpha=30^{\circ}$, the superposition of a short and long wavelength instability is sustained up to approximately $0.8 \mathrm{~s}$. Afterwards, the film reorganizes into well-separated rivulets. By increasing the inclination angle further, the dominant role of short-wave capillary instabilities vanishes and initial spanwise structuring arises with a wavelength associated with the destabilization of the main wave hump. 

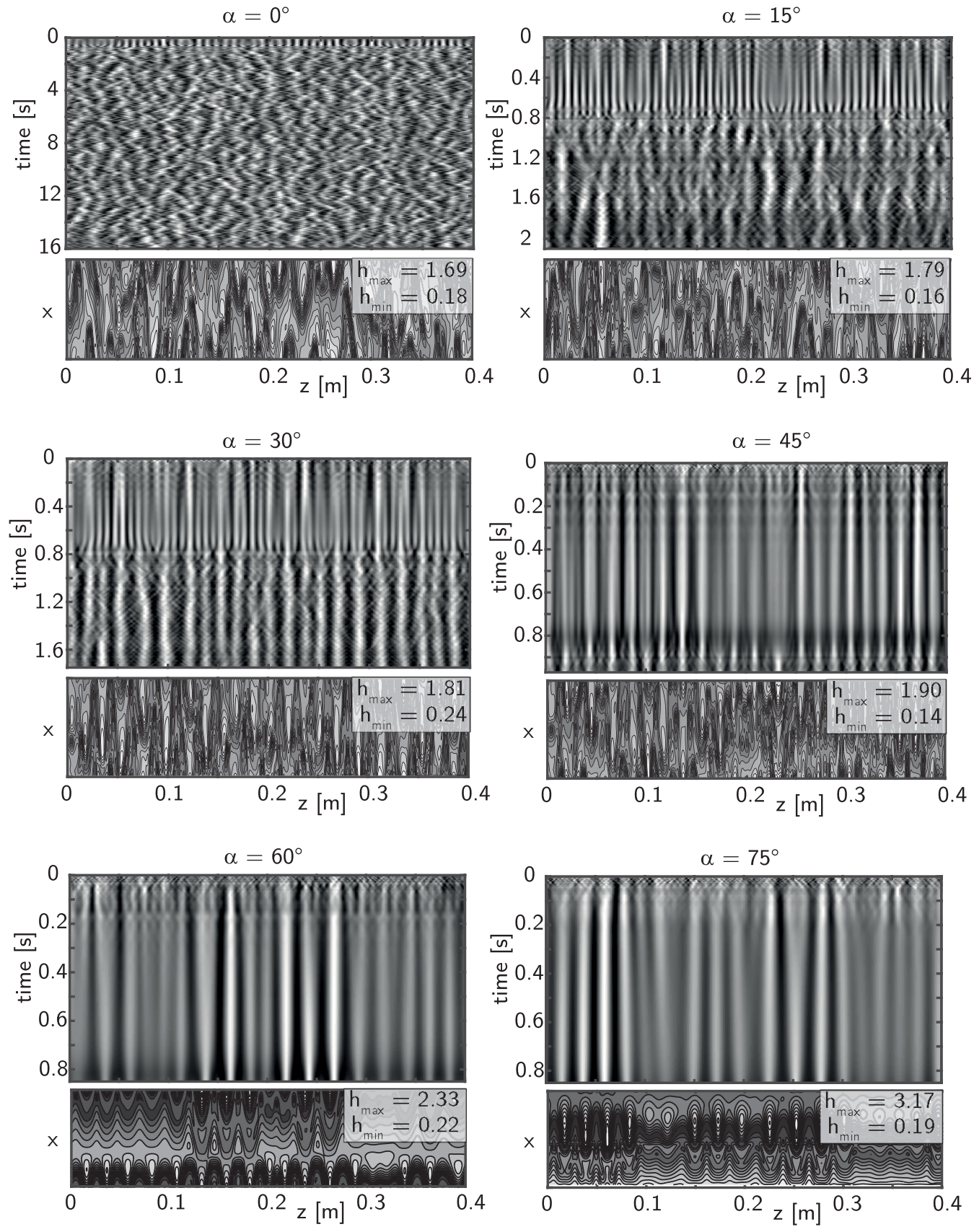

FIG. 17. Temporal plots of the average spanwise film height profile $\bar{h}_{\mathrm{z} \text {,norm }}(t, z)$ and spatial plots of the last simulated time step for case II employing IC 3 . Dark to light colors span the value range $[0,1]$ of the normalized mean spanwise film topology [see Eq. (14)]. Spatial plots of the last time step are normalized by the minimum and maximum film height. 


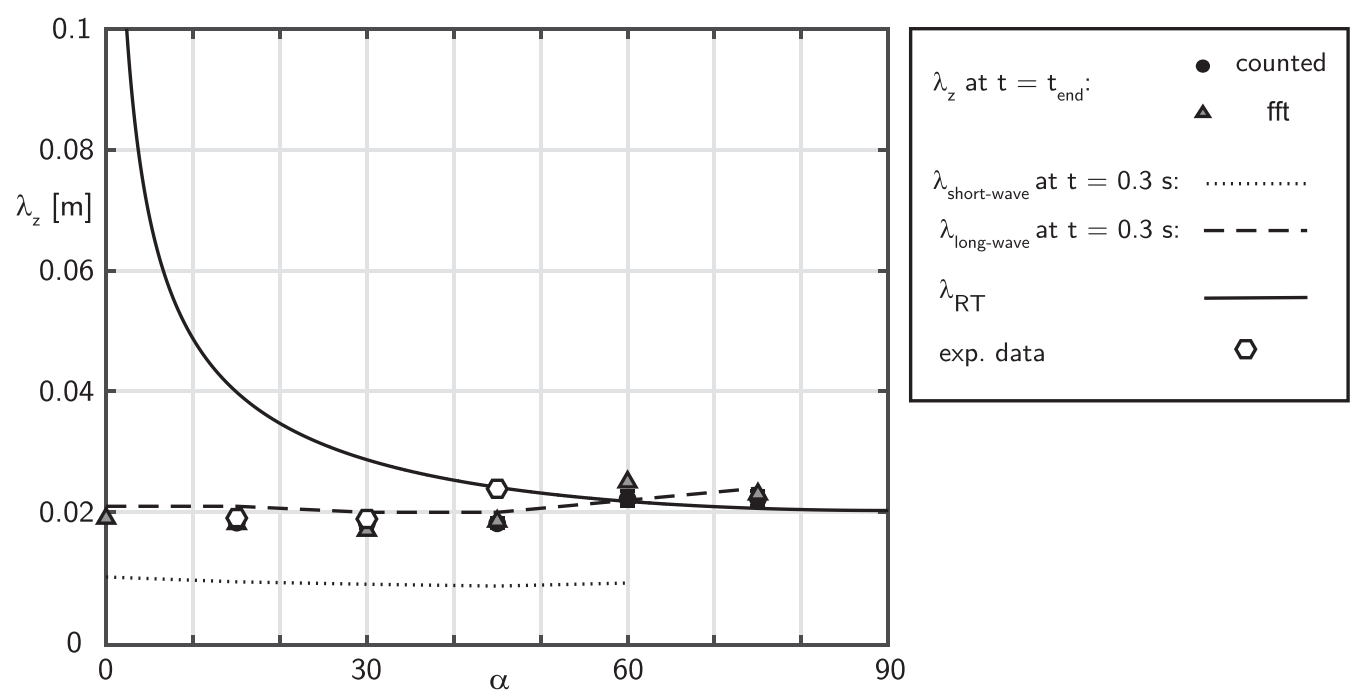

FIG. 18. Quantitative evaluation of the wavelength of spanwise structuring (rivulet formation) for case II under variation of wall inclination. The most amplified wavelength of the primary RT instability as a function of the inclination angle (continuous line) is indicated. Adding to this, most amplified wavelengths (short-wave and long-wave modes) determined from the growth rate spectrum of spanwise perturbations at $t=0.3 \mathrm{~s}$, i.e., within the linear growth phase (dashed and dotted line) are shown. Experimental data points taken from [11].

\section{Wavelength of spanwise structures}

A quantification of observed spanwise wavelengths is conducted analogously to case I and shown in Fig. 18. Dominant spanwise wavelengths at the end of the film evolution are compared to the initially most amplified spanwise wavelengths determined from the growth rate spectrum of the average spanwise wave-height profile [see Fig. 16(b)]. In this context, wavelengths corresponding to the short-wave mode ( $\lambda_{\text {short-wave }}$, dotted line) and to the long-wave mode ( $\lambda_{\text {long-wave }}$, dashed line) are shown. Additionally, the wavelength of spanwise structuring associated with the primary RT mechanism [Eq. (15)] is plotted. Most notably, the primary RT mechanism [Eq. (15)] completely fails to predict final rivulet wavelengths except for large inclinations from the vertical $\left(\alpha>45^{\circ}\right)$, for which the wavelengths approach a value of about $0.02 \mathrm{~m}$. As already observed for case I, the best predictor for the characteristics of long-term spanwise structuring is given through maximally amplified wavelengths in the initial stage of film evolution, associated with the destabilization of 2D wavefronts. Specifically, for case II, wavelengths of the long-wave mode ( $\left.\lambda_{\text {long-wave }}\right)$ are seen to accurately capture rivulet wavelengths at the end of simulations $\left(t=t_{\text {end }}\right)$. Interestingly, the short-wave mode is not sustained after destabilization of wavefronts. The results agree well with available experimental data (hexagonal markers in Fig. 18), indicating that the underlying physical mechanisms can be accurately recovered through the performed simulations. Particularly, the large deviation of observed final spanwise wavelengths from the prediction of the RT mechanism is rationalized.

\section{Parameter variation at a constant angle of inclination $\left(\alpha=15^{\circ}, \mathrm{Re}=40, \mathrm{Ka}_{\alpha=0^{\circ}}=13-1000\right)$}

Regarding case I, the angle-dependent formulation of the RT instability $\left[\lambda_{\mathrm{RT}}\right.$, Eq. (15)] was shown to capture the final wavelength of spanwise structuring with satisfying accuracy, at least for inclination angles $\alpha>5^{\circ}$. This was shown to be true for different imposed initial conditions despite possible transient variations in spanwise wavelength. 
At the same time, significant deviations between observed spanwise wavelengths and $\lambda_{\mathrm{RT}}$ have been found for case II, particularly for inclination angles $\alpha<60^{\circ}$. These deviations could be traced back to the growth rate spectrum of spanwise wavelengths associated with the destabilization of wavefronts, which differs significantly from the spectrum associated with the primary RT instability and includes the onset of a short-wave instability of capillary waves preceding the main wave hump. These findings demonstrate that the RT mechanism is not suited for a general description of rivulet formation in suspended falling films. To track the influence of increased capillary forces on rivulet evolution (as present for case II), the Kapitza number (scaling capillary forces to inertial and viscous forces) is varied for a constant angle of inclination $\left(\alpha=15^{\circ}\right)$ and a constant Reynolds number $(\operatorname{Re}=40)$. To this end, Ka has been adjusted by variation of the fluid viscosity according to

$$
v=\left(\frac{\mathrm{Ka}_{\alpha=0^{\circ}} \rho g^{1 / 3}}{\sigma}\right)^{-3 / 4} .
$$

This allows for a direct comparison of simulation results regarding spanwise structuring, because the Kapitza number is varied without changing $l_{c}$ and thus $\lambda_{\mathrm{RT}}$, which are independent of $v$. Note that the imposed flow rate has been adjusted accordingly to keep the Reynolds number constant $(\operatorname{Re}=40)$.

Strikingly, a strong dependence of the evolution of spanwise structures on the Kapitza number is found, which is illustrated in Fig. 19. In this context, simulation results are illustrated analogously to Figs. 9 to 11 and 17. Increasing Ka, well-separated rivulets aligned in flow direction evolve into less pronounced and less aligned rivulet structures with decreased wavelength. Additionally, a regime of short wavelength capillary instabilities dominates the beginning of film evolution for higher Kapitza numbers. For Kapitza numbers 125, 200, and 1000 this is visible through a highfrequency modulation of the average spanwise film height profile (see Fig. 19).

A quantitative evaluation of spanwise wavelengths is illustrated in Fig. 20. Note that values for $\mathrm{Ka}_{\alpha=0^{\circ}}=1000$ should be treated with caution, because of the observed complex film topology with weak alignment of spanwise structures. Nevertheless, a general trend of the wavelength to decrease further might be noted. In fact, the observed rivulet wavelength changes from about $0.02 \mathrm{~m}$ (as observed for case II), to slightly above $0.04 \mathrm{~m}$ (as observed for case I), if $\mathrm{Ka}$ is lowered from a value of 330 to 13.1. Interestingly, for $\mathrm{Ka}_{\alpha=0^{\circ}}=13.1$ the spanwise wavelength found for case $\mathrm{I}$ is recovered despite an increased Reynolds number (from 1 to 40). Again, it is confirmed that the final wavelength of spanwise structuring is well approximated by the maximally amplified spanwise wavelength associated with the destabilization of the main wave hump (dashed line). How the growth rate spectrum changes in variation of Kapitza number is depicted in Fig. 21 (top). Increasing the Kapitza number, a shift of the most amplified long-wave mode to shorter wavelengths as well as the inception of a short-wave mode corresponding to the destabilization of the preceding capillary wave is observed, which indicates the strong dependence of the respective spectrum on the wave topology. Profiles of the associated 2D wavefronts before spanwise destabilization are illustrated at the bottom of Fig. 21. Note that corresponding streamwise wavelengths decrease for an increasing Kapitza number due to a decreased streamwise extend of the simulation domain, which is based on the most amplified streamwise wavelength for any given condition. The elaborated observations have several important implications.

First, the prediction of long-term spanwise structuring in suspended films has to account for the topology of developing 2D wavefronts, which have a significant impact on the growth rate spectrum of spanwise perturbations. This finding is coupled to the precondition that no significant spanwise structuring has taken place before the formation of these wavefronts. However, this is typically the case in naturally developing films where the growth of streamwise perturbations exceeds the growth of spanwise perturbations. Accordingly, emergence of 2D waves precedes the occurrence of spanwise structuring in the form of rivulets. Note that respective growth rates of streamwise and spanwise perturbations should become comparable for large inclinations from the vertical (e.g., 

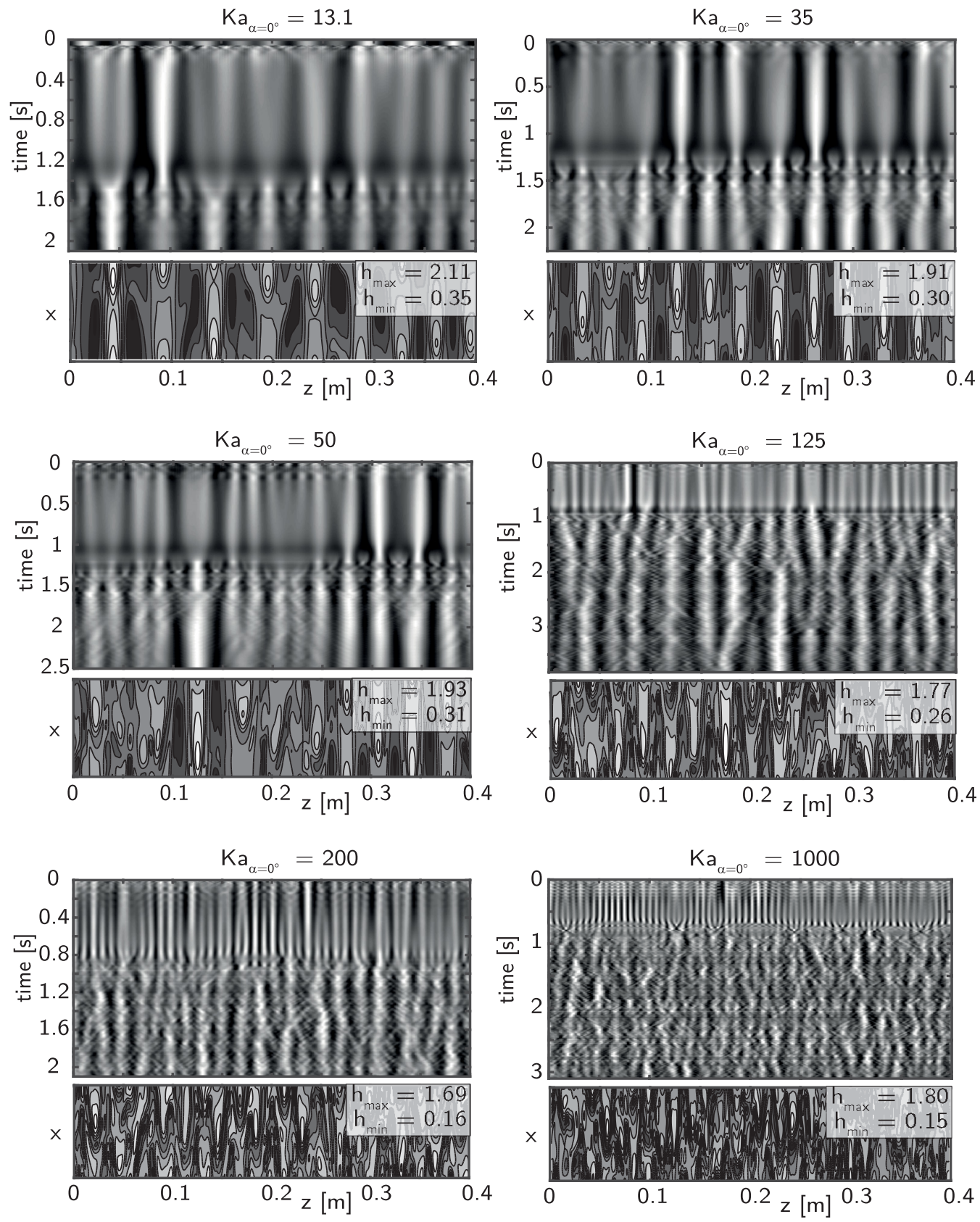

FIG. 19. Variation of the Kapitza number for a constant angle of inclination $\left(\alpha=15^{\circ}\right)$, a constant Reynolds number $(\operatorname{Re}=40)$, and employing IC3: Temporal plots of the average spanwise film height profile and spatial plots of the last simulated time step. Dark to light colors span the value range [0,1] of the normalized mean spanwise film topology [see Eq. (14)]. Spatial plots of the last time step are normalized through the minimum and maximum film height. 


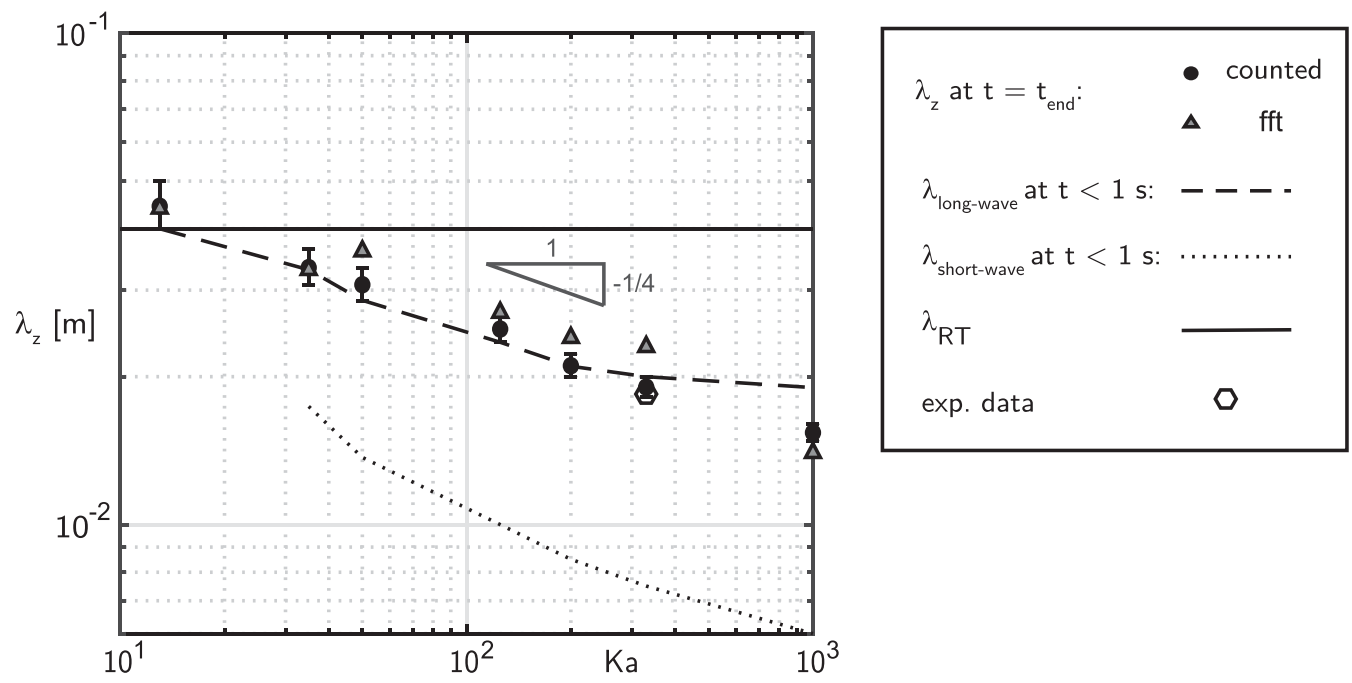

FIG. 20. Rivulet wavelengths for $\mathrm{Ka}_{\alpha=0^{\circ}}=13-1000, \mathrm{Re}=40$ and a constant inclination angle of $\alpha=$ $15^{\circ}$. The most amplified wavelength of the primary RT instability for $\alpha=15^{\circ}$ (continuous line) is indicated. Adding to this, most amplified wavelengths (long-wave and short-wave mode) determined from the growth rate spectrum of spanwise perturbations at $t<1 \mathrm{~s}$, i.e., within the linear growth phase (dashed line) is shown. Experimental data points taken from [11].
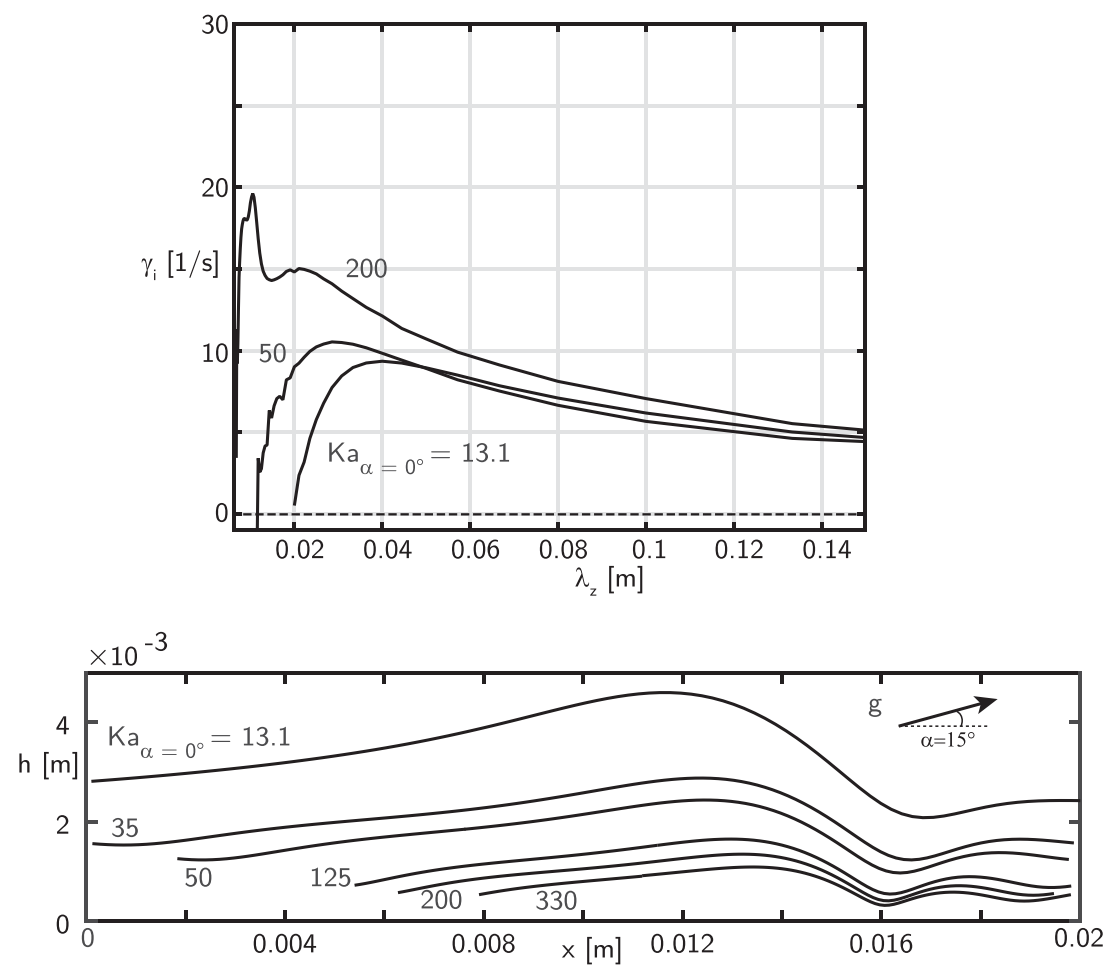

FIG. 21. Linear growth rate spectrum of spanwise perturbations at $t<1 \mathrm{~s}$ for an increasing Kapitza number (top) and profiles of corresponding 2D wavefronts (bottom) for a constant Reynolds number ( $\operatorname{Re}=40$ ). 
$\alpha>\alpha_{c}$ ). However, these cases are not regarded within the present numerical analysis because of the possible dripping issue.

Second, we observe the tendency of dominant capillary forces (high $\mathrm{Ka}$ ) to counteract the formation of well-aligned permanent rivulets. Comparing cases I and II, this has been most prominently observed for a vertical inclination. For case I, maintained spanwise structuring could be traced back to dominant waves, which maintain their spanwise position over extended periods of time. At the same time, for the vertical wall case of case II, spanwise meandering of waves without maintained alignment has been observed. Figure 19 illustrates that this remains true at least for small inclinations from the vertical $\left(\alpha=15^{\circ}\right)$ for large enough Ka. Suppressed alignment into rivulets and simultaneously increased spanwise redistribution of fluid due to capillary action might be a dominant mechanism suppressing nonlinear dripping in high Kapitza number fluids like water.

\section{CONCLUSION}

Simulations of suspended falling liquid films have been performed employing the weighted residual integral boundary layer model of Ruyer-Quil and Manneville [38] and its respective computational implementation within the software package WAVEMAKER [31]. In particular, the film evolution has been analyzed regarding spanwise structuring and rivulet formation due to the destabilizing action of gravity for two distinct cases and under variation of numerically imposed initial conditions. Specific focus was on the wavelength selection mechanisms of rivulet formation and the evolution of their wavelength over time. The two investigated cases are a low Reynolds and Kapitza numbers case (case I: $\operatorname{Re}=1, \mathrm{Ka}_{\alpha=0^{\circ}}=13.1$ ) and an intermediate Reynolds and Kapitza numbers case (case II: $\mathrm{Re}=40, \mathrm{Ka}_{\alpha=0^{\circ}}=330$ ) for both of which experimental data were recently provided in the study of Charogiannis et al. [11]. The parameter space used in the simulations extends around available experimental data points and considers inclination angles of the bounding wall between $\alpha=0^{\circ}$ and $\alpha=75^{\circ}$.

Experimental results could be recovered with satisfying accuracy for both cases indicating the validity of employed numerical methods for the problem in question. Specifically, in agreement with experimental data, it has been observed that an inclination angle-dependent formulation of the RT mechanism $\left(\lambda_{\mathrm{RT}}\right)$ satisfyingly describes the wavelength of evolving rivulets for sufficiently low Re and Ka numbers (case I) for $\alpha>5^{\circ}$. This was shown for varying types and magnitudes of initially imposed noise. However, it has been observed that an asynchronous destabilization of consecutive wavefronts might lead to decreased spanwise wavelengths during the early stages of film evolution. Adding to this, the primary RT mechanism was shown to fail to predict spanwise structuring for inclination angles near the vertical $\left(\alpha<5^{\circ}\right)$. Analysis of the growth rate spectrum of spanwise perturbations associated with the destabilization of wavefronts was shown to provide the best predictor for long-term film evolution.

For sufficiently moderate Re and Ka numbers (case II), and again in accordance with experimental findings, a significant deviation between the prediction of $\lambda_{\mathrm{RT}}$ and numerical results has been observed. Furthermore, a competing short-wave instability of capillary origin associated with waves preceding the main wave hump was shown to impact early spanwise structuring. The influence of capillary instabilities on rivulet evolution has been elucidated through variation of $\mathrm{Ka}$ at a constant inclination of $\alpha=15^{\circ}$. The linear growth rate spectrum of spanwise perturbations of destabilizing 2D wavefronts exhibits a strong dependence on $\mathrm{Ka}$ and the wavelength of spanwise structuring matches well with the most amplified wavelength of respective spectra. For large enough Ka, suppressed alignment into rivulets and simultaneously increased spanwise redistribution of fluid is observed, which might be a dominant mechanism suppressing nonlinear dripping in high Kapitza number fluids like water.

For all investigated cases, it has been revealed that the final wavelength of spanwise structuring is best approximated by the maximally amplified spanwise wavelength associated with the linear spanwise destabilization of the main wave hump. 


\section{ACKNOWLEDGMENTS}

Funding for W.R. and R.K. was provided by DFG (Deutsche Forschungsgemeinschaft) under reference number 418676633. B.S. thanks the F.R.S.-FNRS for financial support.

\section{APPENDIX A: DISPERSION RELATION OF THE FULL WRIBL MODEL}

The linear dispersion relation corresponding to the full second-order (WRIBL) model introduced by Ruyer-Quil et al. [29,38] is taken from the monograph by Kalliadasis et al. [3]. The complete expression of the full second-order model is additionally found in recent publications [10,39] and not explicitly shown here. We consider normal mode perturbations around the dimensionless Nusselt flat film solution in the form:

$$
\begin{aligned}
& h=1+a e^{i(k x-\gamma t),} \\
& q=\frac{1}{3}+b e^{i(k x-\gamma t),}
\end{aligned}
$$

where $k=k_{r}+i k_{i}$ and $\gamma=\gamma_{r}+i \gamma_{i}$ are dimensionless complex wave number and dimensionless complex angular frequency, $a, b$ are amplitudes of the perturbation, and $h, q$ are dimensionless film thickness and flow rate. Inserting Eqs. (A1) and (A2) into the full model, linearizing for small amplitudes $a, b$, and writing the expression in Nusselt scaling with characteristic dimensionless groups Reynolds number (Re), Weber number (We), and inclination number $(\mathrm{Ct})$ lead to the dispersion relation

$$
0=A+B k+C k^{2}+D k^{3}+E k^{4}+F k^{5}+G k^{6},
$$

with

$$
\begin{aligned}
A= & 3 i \gamma+\frac{54}{13} \operatorname{Re} \gamma^{2}-\frac{90}{143} i \operatorname{Re}^{2} \gamma^{3}-\frac{12}{715} \operatorname{Re}^{3} \gamma^{4} \\
B= & -3 i-\frac{522}{143} \operatorname{Re} \gamma+\frac{98}{143} i \operatorname{Re}^{2} \gamma^{2}+\frac{108}{5005} \operatorname{Re}^{3} \gamma^{3}, \\
C= & -\mathrm{Ct}+\frac{498}{715} \operatorname{Re}+\gamma\left(\frac{27}{5} i+\frac{12}{65} i \mathrm{CtRe}-\frac{2936}{13013} i \operatorname{Re}^{2}\right) \\
& +\gamma^{2}\left(\frac{3231}{3640} \operatorname{Re}+\frac{27}{5005} \mathrm{CtRe}^{2}-\frac{612}{65065} \operatorname{Re}^{3}\right)-\frac{2027}{80080} i \operatorname{Re}^{2} \gamma^{3}, \\
D= & -\frac{12}{5} i-\frac{304}{5005} i \mathrm{Ct} \operatorname{Re}+\frac{1368}{65065} i \operatorname{Re}^{2}+\gamma\left(-\frac{2441}{4004} \operatorname{Re}-\frac{16}{5005} \mathrm{CtRe}^{2}+\frac{1104}{715715} \operatorname{Re}^{3}\right) \\
& +\frac{3439}{145600} i \operatorname{Re}^{2} \gamma^{2}, \\
E= & \frac{30993}{320320} \operatorname{Re}^{2}+\frac{148}{325325} \mathrm{CtRe}^{2}-\frac{48}{715715} \operatorname{Re}^{3}-\mathrm{We}^{2} \\
& +\gamma\left(-\frac{4591}{650650} i \operatorname{Re}^{2}+\frac{12}{65} i \mathrm{WeRe}\right)+\frac{27}{5005} \mathrm{WeRe}^{2} \gamma^{2}, \\
F= & \frac{1773}{2602600} i \operatorname{Re}^{2}-\frac{304}{5005} i \mathrm{We} \operatorname{Re}-\frac{16}{5005} \mathrm{WeRe}^{2} \gamma, \\
G= & \frac{148}{325325} \mathrm{WeRe}^{2} .
\end{aligned}
$$



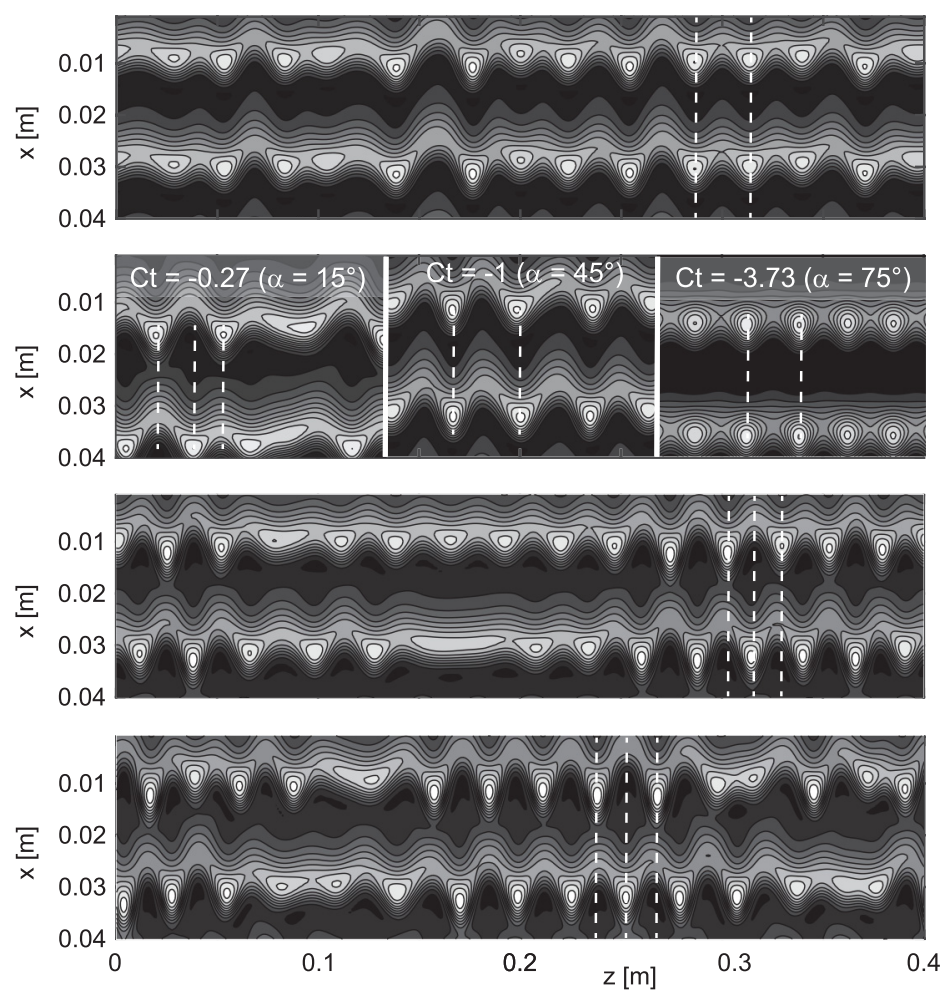

spanwise noise: $a_{\text {span }}=0.01$

2D noise: $\quad a_{2 D}=0.0001$

synchronous destabilization of $2 \mathrm{D}$ waves

spanwise noise: $\quad a_{\text {span }}=0.001$

2D noise:

$a_{2 D}=0.0001$

asynch. | synch. | synch. destabilization of $2 \mathrm{D}$ waves

spanwise noise: $\quad a_{\text {span }}=0.0001$

2D noise:

$a_{2 D}=0.0001$

asynchronous destabilization of $2 \mathrm{D}$ waves

spanwise noise: $a_{\text {span }}=0.001$

2D noise: $\quad a_{2 D}=0.001$

asynchronous destabilization of $2 \mathrm{D}$ waves

FIG. 22. Transition from synchronous to asynchronous destabilization of 2D waves depending on the magnitude of imposed spanwise noise and wall inclination. Conditions: $\operatorname{Re}=1 ; \mathrm{Ka}=13.1 ; \mathrm{Ct}=-1\left(\alpha=45^{\circ}\right)$ or as labeled. Initial condition of the film surface is given by $h^{*}$ [see Eq. (B1)], with indicated relative noise amplitudes.

\section{APPENDIX B: SYNCHRONOUS VERSUS ASYNCHRONOUS DESTABILIZATION OF CONSECUTIVE WAVEFRONTS}

Figure 22 provides a parameter variation of imposed initial noise to address the selection mechanism between synchronous and asynchronous patterns. For this purpose, an additional systematic spanwise noise that is constant in the $x$ direction is added to the noise already imposed in IC1. The varied initial condition is given by

$$
h^{*}=h_{\mathrm{IC} 1}(x, z)+h_{\mathrm{span}}(z),
$$

with

$$
h_{\mathrm{ICl} 1}(x, z)=1+a_{2 \mathrm{D}} \operatorname{rand}(x, z)+0.5 \sin \left(\frac{x}{\lambda_{x, \max }} 2 \pi\right)
$$

and

$$
h_{\text {span }}(z)=a_{\text {span }} \operatorname{rand}(z),
$$

where $a_{2 \mathrm{D}}$ and $a_{\text {span }}$ define noise amplitudes. Note that this type of additional noise can be viewed as small persistent perturbations in the distribution blade of the experimental setup or small modulations of the substrate surface.

If the spanwise noise exceeds the initial 2D noise by at least one order of magnitude, a synchronous destabilization is observed. Contrarily, an asynchronous destabilization is sustained 
if the spanwise noise is of the same order as the $2 \mathrm{D}$ noise or lower. At the same time, a transition between synchronous and asynchronous wave fronts is also observed by variation of wall inclination (varying Ct) as illustrated in the second row of Fig. 22. Specifically, a transition from a synchronous to an asynchronous destabilization can be induced by lowering the value of $\mathrm{Ct}$, i.e., lowering the influence of the RT instability. The given results illustrate the sensitivity of the mode selection (synchronous versus asynchronous) depending on the imposed initial conditions, as was already shown in Scheid et al. [30]. Hence, caution has to be exercised in interpreting related experimental data where imposed noise at film initialization is generally not or hardly quantifiable.

[1] S. V. Alekseenko, V. E. Nakoryakov, and B. G. Pokusaev, in Wave Flow of Liquid Films, edited by T. Fukano (Begell House, New York, 1994).

[2] H.-C. Chang and E. Demekhin, in Complex Wave Dynamics on Thin Films, edited by D. Möbius and R. Miller (Elsevier, Amsterdam, 2002).

[3] S. Kalliadasis, C. Ruyer-Quil, B. Scheid, and M. Velarde, Falling Liquid Films (Springer-Verlag, London, 2012).

[4] F. F. Abdelall, S. I. Abdel-Khalik, D. L. Sadowski, S. Shin, and M. Yoda, On the Rayleigh-Taylor instability for confined liquid films with injection through the bounding surfaces, Int. J. Heat Mass Transf. 49, 1529 (2006).

[5] L. A. Booth, Central station power generation by laser-driven fusion, Nucl. Eng. Des. 24, 263 (1973).

[6] J. Williams, T. Merson, F. Finch, F. Schilling, and T. Frank, A conceptual laser controlled thermonuclear reactor power plant, in Proceedings First Topical Meeting on the Technology of Controlled Fusion (San Diego, California, 1974).

[7] G. Balestra, P.-T. Brun, and F. Gallaire, Rayleigh-Taylor instability under curved substrates: An optimal transient growth analysis, Phys. Rev. Fluids 1, 083902 (2016).

[8] A. Lee, P.-T. Brun, J. Marthelot, G. Balestra, F. Gallaire, and P. M. Reis, Fabrication of slender elastic shells by the coating of curved surfaces, Nat. Commun. 7, 11155 (2016).

[9] A. Indeikina, I. Veretennikov, and H.-C. Chang, Drop fall-off from pendent rivulets, J. Fluid Mech. 338, 173 (1997).

[10] B. Scheid, N. Kofman, and W. Rohlfs, Critical inclination for absolute/convective instability transition in inverted falling films, Phys. Fluids 28, 044107 (2016).

[11] A. Charogiannis, F. Denner, B. G. M. van Wachem, S. Kalliadasis, B. Scheid, and C. N. Markides, Experimental investigations of liquid falling films flowing under an inclined planar substrate, Phys. Rev. Fluids 3, 114002 (2018).

[12] A. J. Babchin, A. L. Frenkel, B. G. Levich, and G. I. Sivashinsky, Nonlinear saturation of Rayleigh-Taylor instability in thin films, Phys. Fluids 26, 3159 (1983).

[13] M. Fermigier, L. Limat, J. E. Wesfreid, P. Boudinet, and C. Quillet, Two-dimensional patterns in RayleighTaylor instability of a thin layer, J. Fluid Mech. 236, 349 (1992).

[14] G. Taylor, The instability of liquid surfaces when accelerated in a direction perpendicular to their planes, Proc. R. Soc. A 201, 192 (1950).

[15] P.-T. Brun, A. Damiano, P. Rieu, G. Balestra, and F. Gallaire, Rayleigh-Taylor instability under an inclined plane, Phys. Fluids 27, 084107 (2015).

[16] M. Rietz, B. Scheid, F. Gallaire, N. Kofman, R. Kneer, and W. Rohlfs, Dynamics of falling films on the outside of a vertical rotating cylinder: Waves, rivulets and dripping transitions. J. Fluid Mech. 832, 189 (2017).

[17] W. Rohlfs, L. M. F. Cammiade, M. Rietz, and B. Scheid, On the effect of electrostatic surface forces on dielectric falling films, J. Fluid Mech. 906, A18 (2021).

[18] V. Lapuerta, F. J. Mancebo, and J. M. Vega, Control of Rayleigh-Taylor instability by vertical vibration in large aspect ratio containers, Phys. Rev. E 64, 016318 (2001). 
[19] A. Alexeev and A. Oron, Suppression of the Rayleigh-Taylor instability of thin liquid films by the Marangoni effect, Phys. Fluids 19, 082101 (2007).

[20] D. E. Weidner, L. W. Schwartz, and M. H. Eres, Suppression and reversal of drop formation in a model paint film, Chem. Prod. Process Model. 2, 1 (2007).

[21] R. Cimpeanu, D. T. Papageorgiou, and P. G. Petropoulos, On the control and suppression of the RayleighTaylor instability using electric fields, Phys. Fluids 26, 022105 (2014).

[22] R. J. Tomlin, R. Cimpeanu, and D. T. Papageorgiou, Instability and dripping of electrified liquid films flowing down inverted substrates, Phys. Rev. Fluids 5, 013703 (2020).

[23] J. Marthelot, E. F. Strong, P. M. Reis, and P.-T. Brun, Designing soft materials with interfacial instabilities in liquid films, Nat. Commun. 9, 4477 (2018).

[24] S. I. Cheng and J. Cordero, Droplet formation from a liquid film over a rotating cylinder, AIAA J. 1, 2597 (1963).

[25] G. Lerisson, P. G. Ledda, G. Balestra, and F. Gallaire, Dripping down the rivulet, Phys. Rev. Fluids 4, 100504 (2019).

[26] P. G. Ledda, G. Lerisson, G. Balestra, and F. Gallaire, Instability of a thin viscous film flowing under an inclined substrate: The emergence and stability of rivulets, J. Fluid Mech. 904, A23 (2020).

[27] G. Lerisson, P. G. Ledda, G. Balestra, and F. Gallaire, Instability of a thin viscous film flowing under an inclined substrate: Steady patterns, J. Fluid Mech. 898, A6 (2020).

[28] G. F. Dietze, W. Rohlfs, K. Nährich, and B. Scheid, Three-dimensional flow structures in laminar falling liquid films, J. Fluid Mech. 743, 75 (2014).

[29] C. Ruyer-Quil and P. Manneville, Improved modeling of flows down inclined planes, Eur. Phys. J. B 15, 357 (2000).

[30] B. Scheid, C. Ruyer-Quil, and P. Manneville, Wave patterns in film flows: Modelling and threedimensional waves, J. Fluid Mech. 562, 183 (2006).

[31] W. Rohlfs, M. Rietz, and B. Scheid, Wavemaker: The three-dimensional wave simulation tool for falling liquid films, SoftwareX 7, 211 (2018).

[32] Y. Y. Trifonov, Bifurcations of two-dimensional into three-dimensional wave regimes for a vertically flowing liquid film, Fluid Dyn. 25, 741 (1990).

[33] H.-C. Chang, M. Cheng, E. A. Demekhin, and D. I. Kopelevich, Secondary and tertiary excitation of three-dimensional patterns on a falling film, J. Fluid Mech. 270, 251 (1994).

[34] J. Liu, J. B. Schneider, and J. P. Gollub, Three-dimensional instabilities of film flows, Phys. Fluids 7, 55 (1995).

[35] S. M. Kharlamov, V. V. Guzanov, A. V. Bobylev, S. V. Alekseenko, and D. M. Markovich, The transition from two-dimensional to three-dimensional waves in falling liquid films: Wave patterns and transverse redistribution of local flow rates, Phys. Fluids 27, 114106 (2015).

[36] N. Kofman, W. Rohlfs, F. Gallaire, B. Scheid, and C. Ruyer-Quil, Prediction of two-dimensional dripping onset of a liquid film under an inclined plane, Int. J. Multiphase Flow 104, 286 (2018).

[37] N. Kofman, S. Mergui, and C. Ruyer-Quil, Three-dimensional instabilities of quasi-solitary waves in a falling liquid film, J. Fluid Mech. 757, 854 (2014).

[38] C. Ruyer-Quil and P. Manneville, Further accuracy and convergence results on the modeling of flows down inclined planes by weighted-residual approximations, Phys. Fluids 14, 170 (2002).

[39] W. Rohlfs, P. Pischke, and B. Scheid, Hydrodynamic waves in films flowing under an inclined plane, Phys. Rev. Fluids 2, 044003 (2017). 